\title{
On the exponential Diophantine equation related to powers of two consecutive terms of Lucas sequences
}

\author{
Mahadi Ddamulira ${ }^{1,2}$ (D) Florian Luca ${ }^{3,4,5}$ \\ Received: 28 January 2020 / Accepted: 30 March 2020 / Published online: 23 July 2020 \\ (c) The Author(s) 2020
}

\begin{abstract}
Let $r \geq 1$ be an integer and $\mathbf{U}:=\left(U_{n}\right)_{n>0}$ be the Lucas sequence given by $U_{0}=0$, $U_{1}=1$, and $U_{n+2}=r U_{n+1}+U_{n}$, for all $n \geq 0$. In this paper, we show that there are no positive integers $r \geq 3, x \neq 2, n \geq 1$ such that $U_{n}^{x}+U_{n+1}^{x}$ is a member of $\mathbf{U}$.
\end{abstract}

Keywords Lucas sequences · Linear forms in logarithms · Baker's method

Mathematics Subject Classification 11B39 $\cdot 11$ D61 $\cdot$ 11J86

\section{Introduction}

Let $r \geq 1$ be an integer and $\mathbf{U}:=\left(U_{n}\right)_{n \geq 0}$ be the Lucas sequence given by $U_{0}=$ $0, U_{1}=1$, and

$$
U_{n+2}=r U_{n+1}+U_{n}
$$

Open access funding provided by Austrian Science Fund (FWF). M. D. was supported by the FWF Projects: F5510-N26-Part of the special research program (SFB), "Quasi Monte Carlo Methods: Theory and Applications" and W1230_- "Doctoral Program Discrete Mathematics”. F. L. was supported by Grant RTNUM19 from CoEMaSS, Wits, South Africa.

$凶$ Mahadi Ddamulira

mddamulira@tugraz.at; mahadi@aims.edu.gh

Florian Luca

Florian.Luca@wits.ac.za

1 Institute of Analysis and Number Theory, Graz University of Technology, Kopernikusgasse 24/II, 8010 Graz, Austria

2 Max Planck Institute for Mathematics, Bonn, Germany

3 School of Mathematics, University of the Witwatersrand, Johannesburg, South Africa

4 Research Group in Algebraic Structures and Applications, King Abdulaziz University, Jeddah, Saudi Arabia

5 Centro de Ciencias Matemáticas UNAM, Morelia, Mexico 
for all $n \geq 0$. When $r=1 \mathbf{U}$ coincides with the Fibonacci sequence while when $r=2$ $\mathbf{U}$ coincides with the Pell sequence. It is well known that

$$
U_{n}^{2}+U_{n+1}^{2}=U_{2 n+1} \text { for all } n \geq 0 \text {. }
$$

In particular, the identity (2) tells us that the sum of the squares of two consecutive terms of $\mathbf{U}$ is also a term of $\mathbf{U}$. When $r=1$, we even have $U_{n}+U_{n+1}=U_{n+2}$ for all $n \geq 0$ since $\mathbf{U}$ is the Fibonacci sequence. We thus consider the Diophantine equation

$$
U_{n}^{x}+U_{n+1}^{x}=U_{m}
$$

in nonnegative integers $(n, m, x)$ which by Eq. (2) has the parametric solution $m=$ $2 n+1$ when $x=2$ for any $r \geq 1$ and even the parametric solution $m=n+2$ when $x=1$ if $r=1$. For $r=1$ Luca and Oyono [8] proved that Eq. (3) has no positive integer solutions $(n, m, x)$ with $n \geq 2$ and $x \geq 3$. Rihane et al. [11] studied Eq. (3) when $r=2$ and proved that there is no positive integer solution $(n, m, x)$ of it with $x \neq 2$. In the same spirit, Gómez Ruiz and Luca [5] studied Eq. (3) with $\mathbf{U}=\left\{F_{n}^{(k)}\right\}_{n \geq-(k-2)}$, which is the $k-$ generalised Fibonacci sequence of recurrence

$$
F_{n}^{(k)}=F_{n-1}^{(k)}+F_{n-2}^{(k)}+\cdots+F_{n-k}^{(k)} \text { for all } n \geq 2
$$

with the initial conditions $F_{-(k-2)}^{(k)}=F_{-(k-2)}^{(k)}=\cdots=F_{0}^{(k)}=0$ and $F_{1}^{(k)}=1$. When $k=2$, this sequence coincides with the sequence of Fibonacci numbers. They proved that Eq. (3) has no positive integer solution $(k, n, m, x)$ with $k \geq 3, n \geq 2$, and $x \geq 1$. Another related result involving the balancing numbers was studied by Rihane et al. in [10].

In this paper, we study Eq. (3) in nonnegative integers $(r, n, m, x)$ treating $r$ as an integer parameter. We may assume that $r \geq 3$ since the cases $r \in\{1,2\}$ have been treated already in $[8,11]$, respectively. The solution with $(n, m)=(0,1)$ (for any $r$ and $x$ ) is obvious so we omit it and suppose that $n$ is positive. Our main result is the following.

Theorem 1 There is no positive integer solution $(r, n, m, x)$ of Diophantine equation (3) with $r \geq 3$ and $x \neq 2$.

\section{Preliminary results}

\subsection{The Lucas sequence}

Let

$$
(\alpha, \beta):=\left(\frac{r+\sqrt{r^{2}+4}}{2}, \frac{r-\sqrt{r^{2}+4}}{2}\right)
$$


be the roots of the characteristic equation $x^{2}-r x-1=0$ of the Lucas sequence $\mathbf{U}=\left(U_{n}\right)_{n \geq 0}$. We put $\Delta=r^{2}+4=(\alpha-\beta)^{2}$ for the discriminant of the above quadratic equation. The Binet formula for the general term of $\mathbf{U}$ is given by

$$
U_{n}:=\frac{\alpha^{n}-\beta^{n}}{\alpha-\beta} \text { for all } n \geq 0 .
$$

One may prove by induction that the inequality

$$
\alpha^{n-2} \leq U_{n} \leq \alpha^{n-1}
$$

holds for all positive integers $n$. It is also easy to show that the inequality

$$
\frac{U_{n}}{U_{n+1}}<\frac{1}{r}
$$

holds for all $n \geq 2$. Indeed, it follows from $U_{n+1}=r U_{n}+U_{n-1}>r U_{n}$ for $n \geq 2$. At one point of the argument we will need the companion Lucas sequence $\mathbf{V}:=\left\{V_{n}\right\}_{n \geq 0}$ given by $V_{0}=2, V_{1}=r$, and $V_{n+2}=r V_{n+1}+V_{n}$ for all $n \geq 0$. Its Binet formula is

$$
V_{n}=\alpha^{n}+\beta^{n} \text { for all } n \geq 0 \text {. }
$$

There are many relations between members of $\mathbf{U}$ and $\mathbf{V}$ such as

$$
\begin{aligned}
U_{2 n} & =U_{n} V_{n} ; \\
U_{n+1}^{2}-U_{n} U_{n+2} & =(-1)^{n}, \\
V_{n}^{2}-\Delta U_{n}^{2} & =4(-1)^{n}, \\
2 U_{m+n} & =U_{m} V_{n}+U_{n} V_{m}, \\
\operatorname{gcd}\left(U_{n}, U_{m}\right) & =U_{\operatorname{gcd}(m, n)} .
\end{aligned}
$$

We record another one. All such identities follow easily from the Binet formulas (4) and (7) of $\mathbf{U}$ and $\mathbf{V}$, respectively.

Lemma 1 If $n$ is odd then

$$
U_{n}-1= \begin{cases}U_{(n-1) / 2} V_{(n+1) / 2} \text { if } n \equiv 1 & (\bmod 4), \\ U_{(n+1) / 2} V_{(n-1) / 2} \text { if } n \equiv 3 & (\bmod 4) .\end{cases}
$$

Both sequences $\mathbf{U}$ and $\mathbf{V}$ can be extended to negative indices either by allowing $n$ to be negative in the Binet formulas (4) and (7) or simply by using the recurrence relation to extend $\mathbf{U}$ to negative indices via $U_{-n}=-r U_{-(n-1)}+U_{-(n-2)}$ for all $n \geq 1$. The same applies to V. All the above formulas given in Eqs. (8), (9), (10), (11), and (13) hold when the indices are arbitrary integers, not necessarily nonnegative.

The following lemma is useful. For further details we refer the reader to the book of Koshy [6]. 
Lemma 2 Let $\left\{U_{n}(r)\right\}_{n \geq 0} \subseteq \mathbf{Z}[r]$ be the sequence of polynomials defined by $U_{0}(r)=$ $0, U_{1}(r)=1$, and

$$
U_{n+2}(r)=r U_{n+1}(r)+U_{n}(r) \text { for all } n \geq 0 .
$$

Then,

$$
U_{n}(r)=\sum_{\substack{0 \leq k \leq n \\
k \neq n}}\left(\begin{array}{c}
\frac{n+k-1}{2} \\
k
\end{array}\right) r^{k}
$$

Note that the summation range above is only over these $k \in[0, n]$ which have different parity than $n$. We record the following easy but useful consequence of the formula (14).

Lemma 3 We have $r \mid U_{n}$ if $n$ is even and $r \mid U_{n}-1$ if $n$ is odd.

\subsection{Logarithmic height}

Let $\eta$ be an algebraic number of degree $d$ with minimal primitive polynomial over the integers

$$
a_{0} x^{d}+a_{1} x^{d-1}+\cdots+a_{d}=a_{0} \prod_{i=1}^{d}\left(x-\eta^{(i)}\right)
$$

where the leading coefficient $a_{0}$ is positive and the $\eta^{(i)}$ 's are the conjugates of $\eta$. The logarithmic height of $\eta$ is given by

$$
h(\eta):=\frac{1}{d}\left(\log a_{0}+\sum_{i=1}^{d} \log \left(\max \left\{\left|\eta^{(i)}\right|, 1\right\}\right)\right) .
$$

In particular, if $\eta=p / q$ is a rational number with $\operatorname{gcd}(p, q)=1$ and $q>0$, then $h(\eta)=\log \max \{|p|, q\}$. The following are some of the properties of the logarithmic height function $h(\cdot)$, which will be used in the next sections of this paper without reference:

$$
\begin{aligned}
h(\eta \pm \gamma) & \leq h(\eta)+h(\gamma)+\log 2, \\
h\left(\eta \gamma^{ \pm 1}\right) & \leq h(\eta)+h(\gamma), \\
h\left(\eta^{s}\right) & =|s| h(\eta) \quad(s \in \mathbb{Z}) .
\end{aligned}
$$

\subsection{Linear forms in logarithms and continued fractions}

The following result on linear forms in three logarithms is due to Mignotte [9]. The result is more general (i.e. the conditions on the parameters involved are somewhat more general), but we will only quote it in the form that we need. 
Theorem 2 Consider three algebraic numbers $\gamma_{1}, \gamma_{2}$, and $\gamma_{3}$, which are all real, greater than 1 and multiplicatively independent. Put

$$
\mathcal{D}:=\left[\mathbb{Q}\left(\gamma_{1}, \gamma_{2}, \gamma_{3}\right): \mathbb{Q}\right]
$$

Let $b_{1}, b_{2}, b_{3}$ be coprime positive integers and consider

$$
\Gamma:=b_{2} \log \gamma_{2}-b_{1} \log \gamma_{1}-b_{3} \log \gamma_{3}
$$

Put

$$
d_{1}:=\operatorname{gcd}\left(b_{1}, b_{2}\right)=\frac{b_{1}}{b_{1}^{\prime}}=\frac{b_{2}}{b_{2}^{\prime}}, \quad d_{3}:=\operatorname{gcd}\left(b_{3}, b_{2}\right)=\frac{b_{2}}{b_{2}^{\prime \prime}}=\frac{b_{3}}{b_{3}^{\prime \prime}} .
$$

Let $A_{1}, A_{2}$, and $A_{3}$ be real numbers such that

$$
A_{i} \geq \max \left\{4,4.296 \log \gamma_{i}+2 \mathcal{D} h\left(\gamma_{i}\right)\right\}, \quad i=1,2,3, \quad \text { and } \Omega:=A_{1} A_{2} A_{3} \geq 100 \text {. }
$$

Put

$$
b^{\prime}:=\left(\frac{b_{1}^{\prime}}{A_{2}}+\frac{b_{2}^{\prime}}{A_{1}}\right)\left(\frac{b_{3}^{\prime \prime}}{A_{2}}+\frac{b_{2}^{\prime \prime}}{A_{3}}\right) \quad \text { and } \log \mathcal{B}:=\max \left\{0.882+\log b^{\prime}, \frac{10}{\mathcal{D}}\right\}
$$

Then, either

$$
\log |\Gamma|>-790.95 \Omega \mathcal{D}^{2}(\log \mathcal{B})^{2}
$$

or one of the following conditions holds:

(i) there exist two positive integers $r_{0}$ and $s_{0}$ such that

$$
r_{0} b_{2}=s_{0} b_{1}
$$

with

$$
r_{0} \leq 5.61 A_{2}(\mathcal{D} \log \mathcal{D})^{\frac{1}{3}} \text { and } s_{0} \leq 5.61 A_{1}(\mathcal{D} \log \mathcal{D})^{\frac{1}{3}}
$$

(ii) there exist integers $r_{1}, s_{1}, t_{1}$, and $t_{2}$, with $r_{1} s_{1} \neq 0$, such that

$$
\left(t_{1} b_{1}+r_{1} b_{3}\right) s_{1}=r_{1} b_{2} t_{2}, \quad \operatorname{gcd}\left(r_{1}, t_{1}\right)=\operatorname{gcd}\left(s_{1}, t_{2}\right)=1
$$

which also satisfy

$$
\begin{aligned}
& \left|r_{1} s_{1}\right| \leq 5.61 \delta A_{3}(\mathcal{D} \log \mathcal{D})^{\frac{1}{3}}, \quad\left|s_{1} t_{1}\right| \leq 5.61 \delta A_{1}(\mathcal{D} \log \mathcal{D})^{\frac{1}{3}} \\
& \left|r_{1} t_{2}\right| \leq 5.61 \delta A_{2}(\mathcal{D} \log \mathcal{D})^{\frac{1}{3}}
\end{aligned}
$$


where

$$
\delta:=\operatorname{gcd}\left(r_{1}, s_{1}\right)
$$

Moreover, when $t_{1}=0$ we can take $r_{1}=1$, and when $t_{2}=0$ we can take $s_{1}=1$.

At some point we will need to treat linear forms in two logarithms of algebraic numbers. To set the stage, let $\gamma_{1}$ and $\gamma_{2}$ be real algebraic numbers which are positive and let $\mathcal{D}:=\left[\mathbb{Q}\left(\gamma_{1}, \gamma_{2}\right): \mathbb{Q}\right]$. Let $b_{1}, b_{2}$ be nonzero integers, let $B_{1}$ and $B_{2}$ be real numbers larger than 1 such that

$$
\log B_{i} \geq \max \left\{h\left(\gamma_{i}\right), \frac{\left|\log \gamma_{i}\right|}{\mathcal{D}}, \frac{1}{\mathcal{D}}\right\}, \quad \text { for } \quad i=1,2
$$

and put

$$
b^{\prime}=\frac{\left|b_{1}\right|}{\mathcal{D} \log B_{2}}+\frac{\left|b_{2}\right|}{\mathcal{D} \log B_{1}} .
$$

Let

$$
\Gamma:=b_{1} \log \gamma_{1}+b_{2} \log \gamma_{2}
$$

The following result of Laurent et al. is Corollary 2 in [7].

Theorem 3 With the above notations assuming furthermore that $\gamma_{1}$ and $\gamma_{2}$ are multiplicatively independent we have

$$
\log |\Gamma|>-24.34 \mathcal{D}^{4}\left(\max \left\{\log b^{\prime}+0.14, \frac{21}{\mathcal{D}}, \frac{1}{2}\right\}\right)^{2} \log B_{1} \log B_{2}
$$

Note that the fact that $\Gamma \neq 0$ is already guaranteed by the condition that $\gamma_{1}$ and $\gamma_{2}$ are multiplicatively independent together with the fact that $b_{1}, b_{2}$ are nonzero integers.

During the calculations we get upper bounds on our variables which are too large, thus we need to reduce them. To do so we use some results from the theory of continued fractions.

For the treatment of linear forms homogeneous in two integer variables we use a well-known classical result in the theory of Diophantine approximation due to Legendre.

Lemma 4 Let $\tau$ be an irrational number, $p_{0} / q_{0}, p_{1} / q_{1}, p_{2} / q_{2}, \ldots$ be the sequence of convergents of the continued fraction expansion of $\tau$ and $M$ be a positive integer. Let $N$ be a nonnegative integer such that $q_{N}>M$. Putting $a(M):=\max \left\{a_{i}: i=\right.$ $0,1,2, \ldots, N\}$ the inequality

$$
\left|\tau-\frac{u}{v}\right|>\frac{1}{(a(M)+2) v^{2}}
$$


holds for all pairs $(u, v)$ of positive integers with $0<v<M$. Furthermore, if

$$
\left|\tau-\frac{u}{v}\right|<\frac{1}{2 v^{2}}
$$

then $u / v=p_{k} / q_{k}$ for some $k \geq 0$.

For a nonhomogeneous linear form in two integer variables we use a slight variation of a result due to Dujella and Pethő (see [4, Lemma 5a]). For a real number $X$, we write $\|X\|:=\min \{|X-n|: n \in \mathbb{Z}\}$ for the distance from $X$ to the nearest integer.

Lemma 5 Let $M$ be a positive integer, $p / q$ be a convergent of the continued fraction expansion of the irrational number $\tau$ such that $q>6 M$, and $A, B, \mu$ be some real numbers with $A>0$ and $B>1$. Furthermore, let $\varepsilon:=\|\mu q\|-M\|\tau q\|$. If $\varepsilon>0$, then there is no solution to the inequality

$$
0<|u \tau-v+\mu|<A B^{-w}
$$

in positive integers $u, v$, and $w$ with

$$
u \leq M \quad \text { and } \quad w \geq \frac{\log (A q / \varepsilon)}{\log B} .
$$

\section{Proof of Theorem 1}

\subsection{The cases $n=1$ or $x=1$}

We assume that $n \geq 1$, as the solution with $n=0$ is trivial. Since $U_{n+1}<U_{n+1}+U_{n}<$ $U_{n+2}$, it follows that the Diophantine equation (3) has no solution with $x=1$. Let us assume that $n=1$. We then get that

$$
U_{m}=1+r^{x}
$$

In particular, $U_{m} \equiv 1(\bmod r)$. Lemma 3 shows that $m \equiv 1(\bmod 2)$ and now Lemma 1 shows that

$$
r^{x}=U_{m}-1=U_{(m-\delta) / 2} V_{(m+\delta) / 2} \quad \text { where } \quad \delta \in\{ \pm 1\}, \quad \delta \equiv m \quad(\bmod 4) .
$$

We now recall the Primitive Divisor Theorem of Carmichael [3] (see [1] for the most general statement) for the sequence $\mathbf{U}$. It states that if $\ell>12$, there is a prime factor $p$ of $U_{\ell}$ which is primitive in the sense that $p \nmid U_{k}$ for any positive $k<\ell$. So, assume $m+\delta \geq 14$. Since $m+\delta$ is even we have $U_{m+\delta}=U_{(m+\delta) / 2} V_{(m+\delta) / 2}$ by the formula (8). Since $U_{m+\delta}$ has a primitive prime factor $p$, the primitive prime $p$ must be a divisor of $V_{(m+\delta) / 2}$, which in turn must divide $r=U_{2}$, a contradiction. Thus, $m+\delta \leq 12$, therefore $m \leq 13$. It thus follows that

$$
r^{x}=U_{m}-1<\alpha^{m-1} \leq \alpha^{12}<\left(r^{2}+4\right)^{6},
$$


so, if $x \geq 13$, then

$$
3^{x-12} \leq r^{x-12}<\left(1+4 / r^{2}\right)^{6} \leq\left(1+4 / 3^{2}\right)^{6}<9.1
$$

which gives $x \leq 14$. Thus, $m \leq 13, x \leq 14$. For each choice of the pair $(m, x)$ with the components in the above ranges, the equation $U_{m}(r)-1-r^{x}=0$ is a polynomial equation in $r$. After a simple computer search, we found no other solutions to Eq. (18) apart from the solution $(n, m, x)=(1,3,2)$ which has $x=2$ so it is part of the parametric family of solutions indicated at Eq. (2).

So, from now on we assume that $n \geq 2$ and $x \geq 3$.

\subsection{Calculations when $n \in[2,100]$ and $x \in[3,100]$}

Using the Eq. (3) and the inequality (5), we get

$$
\alpha^{(n-1) x}<U_{n+1}^{x}<U_{n}^{x}+U_{n+1}^{x}=U_{m} \leq \alpha^{m-1},
$$

and

$$
\alpha^{m-2}<U_{m}=U_{n}^{x}+U_{n+1}^{x}<\left(U_{n}+U_{n+1}\right)^{x}<U_{n+2}^{x}<\alpha^{(n+1) x} .
$$

From the above inequalities, we get the following result which we record for future reference.

Lemma 6 The inequalities

$$
(n-1) x+1<m<(n+1) x+2
$$

hold for all $x \geq 3$ and $n \geq 2$.

We next consider Eq. (14) given in Lemma 2. We write Eq. (3) as

$\left(\sum_{\substack{0 \leq k \leq n \\ k \neq n(\bmod 2)}}\left(\begin{array}{c}\frac{n+k-1}{2} \\ k\end{array}\right) r^{k}\right)^{x}+\left(\sum_{\substack{0 \leq k \leq n+1 \\ k \neq n+1(\bmod 2)}}\left(\begin{array}{c}\frac{n+k}{2} \\ k\end{array}\right) r^{k}\right)^{x}=\sum_{\substack{0 \leq k \leq m \\ k \neq m(\bmod 2)}}\left(\begin{array}{c}\frac{m+k-1}{2} \\ k\end{array}\right) r^{k}$.

Assume first that $n$ is even. Then Eq. (20) becomes

$$
\begin{aligned}
& \left(\frac{n}{2} r+\left(\begin{array}{c}
\frac{n+2}{2} \\
3
\end{array}\right) r^{3}+\cdots\right)^{x}+\left(1+\left(\begin{array}{c}
\frac{n+2}{2} \\
2
\end{array}\right) r^{2}+\left(\begin{array}{c}
\frac{n+4}{2} \\
4
\end{array}\right) r^{4}+\cdots\right)^{x} \\
& =1+\left(\begin{array}{c}
\frac{m+1}{2} \\
2
\end{array}\right) r^{2}+\left(\begin{array}{c}
\frac{m+3}{2} \\
3
\end{array}\right) r^{4}+\cdots,
\end{aligned}
$$


which is equivalent to

$$
\left(\begin{array}{c}
\frac{n+2}{2} \\
2
\end{array}\right) r^{2} x+\left(\begin{array}{c}
\frac{n+4}{2} \\
4
\end{array}\right) r^{4} x+\cdots \equiv\left(\begin{array}{c}
\frac{m+1}{2} \\
2
\end{array}\right) r^{2}+\left(\begin{array}{c}
\frac{m+3}{2} \\
3
\end{array}\right) r^{4}+\cdots\left(\bmod r^{x}\right) .
$$

The above relation implies that

$$
r^{\min \{x, 4\}-2} \mid\left(\begin{array}{c}
\frac{m+1}{2} \\
2
\end{array}\right)-x\left(\begin{array}{c}
\frac{n+2}{2} \\
2
\end{array}\right) .
$$

Similarly, when $n$ is odd, one is led to the analogous divisibility relation

$$
r^{\min \{x, 4\}-2} \mid\left(\begin{array}{c}
\frac{m+1}{2} \\
2
\end{array}\right)-x\left(\begin{array}{c}
\frac{n+1}{2} \\
2
\end{array}\right) .
$$

So, fixing $n \in[2,100]$ and $x \in[3,100]$, inequalities (19) give some range for $m$. For each $(n, x, m)$, divisibility relations (22) and (23) (according to whether $n$ is even or odd) give us some possibilities for $r \geq 3$ and now one checks whether relation (3) holds for this candidate $(n, x, m, r)$. A computer search with Mathematica in this range for $n$ and $x$ which ran for a few hours found no solutions. For the search we didn't actually checked that formula (20) holds but we checked that Eq. (20) does not hold modulo $T$, where $T$ is the product of the first 20 primes. The Mathematica function powermod allowed us to compute the powers of $r$ modulo $T$ arising from the binomial formula rather quickly.

From now on, we assume that $n \geq 2, x \geq 3$ and $\max \{n, x\}>100$.

\subsection{A small linear form in three logs}

We rewrite Eq. (3) as

$$
\frac{\alpha^{m}}{\alpha-\beta}-U_{n+1}^{x}=U_{n}^{x}+\frac{\beta^{m}}{\alpha-\beta} .
$$

Dividing both sides of the above equation by $U_{n+1}^{x}$ and using the inequality (6), we obtain

$$
\left|\alpha^{m}(\alpha-\beta)^{-1} U_{n+1}^{-x}-1\right|=\left(\frac{U_{n}}{U_{n+1}}\right)^{x}+\frac{\beta^{m}}{(\alpha-\beta) U_{n+1}^{x}}<2\left(\frac{U_{n}}{U_{n+1}}\right)^{x}<\frac{2}{r^{x}} .
$$

Put

$$
\Lambda:=\alpha^{m}(\alpha-\beta)^{-1} U_{n+1}^{-x}-1 \text { and } \Gamma:=m \log \alpha-\log (\alpha-\beta)-x \log U_{n+1} .
$$


We observe that $\Lambda=e^{\Gamma}-1$, where $\Lambda$ and $\Gamma$ are given by (26). Since $|\Lambda| \leq 2 / 27$, we have that $e^{|\Gamma|} \leq 27 / 25$ and using the inequality (25) we obtain

$$
|\Gamma|=\left|m \log \alpha-\log \left(\sqrt{r^{2}+4}\right)-x \log U_{n+1}\right| \leq e^{|\Gamma|}\left|e^{|\Gamma|}-1\right| \leq \frac{27|\Lambda|}{25}<\frac{2.2}{r^{x}} .
$$

We record the above inequality for future reference.

Lemma 7 With $\Gamma$ given by formula (26), inequality (27) holds.

We want to apply Theorem 2 with the following data:

$$
\gamma_{1}:=\alpha-\beta=\sqrt{r^{2}+4}, \quad \gamma_{2}:=\alpha, \quad \gamma_{3}:=U_{n+1}, \quad b_{1}:=1, \quad b_{2}:=m, \quad b_{3}:=x .
$$

We need to check that $\gamma_{1}, \gamma_{2}$, and $\gamma_{3}$ are multiplicatively independent. This we do in the next subsection.

\subsection{Checking that $\gamma_{1}, \gamma_{2}, \gamma_{3}$ are multiplicatively independent}

Well, assume they are not and let $i, j, k$ be integers not all zero such that

$$
\gamma_{1}^{i} \gamma_{2}^{j} \gamma_{3}^{k}=1
$$

Squaring and rearranging the above relation we get $\gamma_{2}^{2 j}=\left(\gamma_{1}^{2}\right)^{-i} \gamma_{3}^{-2 k} \in \mathbb{Q}$. However, $\gamma_{2}^{2 j}$ is also a unit, so an algebraic integer whose reciprocal is also an algebraic integer, and it is also positive, so it must be 1 . Thus, $j=0$. It now follows that $i$ and $k$ are both nonzero (since if one of them is, so is the other one) and further $\gamma_{3}=\gamma_{1}^{-i / k}$. In particular, all prime factors of $U_{n+1}$ are prime factors of $\Delta:=r^{2}+4$. But this is also contemplated by the Primitive Divisor Theorem of Carmichael since primes dividing $\Delta$ are not considered primitive. In particular, $U_{n+1}$ does not have primitive prime factors so $n+1 \leq 12$. In fact, Theorem $\mathrm{C}$ in [1] together with Table 1 there show that either $n+1 \in\{2,3,4,6\}$ or $n+1 \in\{5,12\}$ but in this last case, the only such Lucas sequence $\mathbf{U}$ for which either one of $U_{5}$ or $U_{12}$ does not have primitive prime factors is the sequence of Fibonacci numbers, which is not our case. Thus, $n+1 \in\{2,3,6\}$. Further, for each prime $p$ let $z(p)$ be the index of appearance of $p$ in $\mathbf{U}$ defined as the smallest positive integer $k$ such that $p \mid U_{k}$. This always exists for our sequence $\mathbf{U}$ since $\alpha$ is a quadratic unit. It has the additional property that if $\ell$ is a positive integer then $p \mid U_{\ell}$ if and only if $z(p) \mid \ell$. It is also well-known and easy to prove that if $p \mid \Delta$, then $z(p)=p$. Since also $z(p) \mid n+1$ and $n+1 \in\{2,3,6\}$, it follows that the only possibilities for $p$ are $p=2,3$. Hence, $r^{2}+4=2^{a} 3^{b}$. However, 3 cannot divide $r^{2}+4$ for any positive integer $r$ (because -4 is not a quasartic residue modulo 3 ), so $b=0$ and $2^{a}=r^{2}+4$. Thus, $r=2 r_{0}$ is even, $a \geq 3$ and the equation simplifies to $2^{a-2}=r_{0}^{2}+1$. Hence, $r_{0}$ is odd, so $r_{0}^{2} \equiv 1(\bmod 8)$, therefore $2 \| r_{0}^{2}+1$, which leads to $a=3, r_{0}=1$, which gives $r=2$, is not our case. Hence, indeed $\gamma_{1}, \gamma_{2}, \gamma_{3}$ are multiplicatively independent. 


\subsection{Applying Theorem 2}

Since $\gamma_{1}, \gamma_{2}, \gamma_{3} \in \mathbb{Q}(\alpha)$, we have $\mathcal{D}=2$. We also have

$$
r<\alpha<\sqrt{r^{2}+4}<r+1
$$

So, we bound the heights of $\alpha$ and $\sqrt{r^{2}+4}$ in terms of $\log (r+1)$. Since

$h\left(\gamma_{1}\right)=h(\alpha-\beta)=\frac{1}{2} \log \left(r^{2}+4\right)<\log (r+1), \quad h\left(\gamma_{2}\right)=\frac{1}{2} \log \alpha<\frac{1}{2} \log (r+1)$,

and

$$
h\left(\gamma_{3}\right)=\log U_{n+1}<\log \alpha^{n}=n \log \alpha<n \log (r+1),
$$

we can take

$$
A_{1}:=8.296 \log (r+1), \quad A_{2}:=6.296 \log (r+1), \quad A_{3}:=8.296 n \log (r+1) .
$$

Thus $\Omega:=A_{1} A_{2} A_{3}>433 n(\log (r+1))^{3}>100$ since $n \geq 2$ and $r \geq 3$. Then,

$$
\begin{aligned}
b^{\prime} & =\left(\frac{1}{6.296 \log (r+1)}+\frac{m}{8.296 \log (r+1)}\right)\left(\frac{x}{6.296 \log (r+1)}+\frac{m}{8.296 n \log (r+1)}\right) \\
& <\frac{m}{(\log (r+1))^{2}}\left(\frac{1}{6.296}+\frac{1}{8.296}\right) \frac{x}{\log (r+1)}\left(\frac{1}{6.296}+\frac{2}{8.296}\right) \\
& <\frac{0.12 m x}{(\log (r+1))^{2}} .
\end{aligned}
$$

In the above chain of inequalities, we used the fact that $m<(n+1) x+2<2 n x$ [see inequality (19)] since $n \geq 2$ and $x \geq 3$. Further, putting

$$
\log \mathcal{B}:=\max \left\{0.882+\log \left(\frac{0.12 m x}{(\log (r+1))^{2}}\right), 5\right\},
$$

we have that either the inequality

$$
\log |\Gamma|>-790.95 \times 434 n(\log (r+1))^{3} \times 2^{2} \times(\log \mathcal{B})^{2}
$$

holds, or the other possibilities (i), (ii) from Theorem 2 hold. We treat (i) and (ii) later and deal with the above inequality (29) at this stage. If $\log \mathcal{B}=5$, then

$$
x<m x<\frac{e^{5-0.882}}{0.12}(\log (r+1))^{2}<512(\log (r+1))^{2} .
$$


On the other hand, if $\log \mathcal{B}>5$, then

$$
\log \mathcal{B}:=0.882+\log \left(\frac{0.12 m x}{(\log (r+1))^{2}}\right)<\log \left(\frac{0.3 m x}{(\log (r+1))^{2}}\right)
$$

where in the above inequality we used the fact that $e^{0.882} \times 0.12<0.3$. Thus, we get that

$$
\begin{aligned}
\log |\Gamma| & >-790.95 \times 434 n(\log (r+1))^{3} \times 2^{2} \times\left(\log \left(\frac{0.3 m x}{(\log (r+1))^{2}}\right)\right)^{2} \\
& >-1.374 \times 10^{6} n(\log (r+1))^{3}\left(\log \left(\frac{0.3 m x}{(\log (r+1))^{2}}\right)\right)^{2} .
\end{aligned}
$$

Comparing this inequality with Eq. (27), we get that

$$
x \log r-\log 2.2<1.374 \times 10^{6} n(\log (r+1))^{3}\left(\log \left(\frac{0.3 m x}{(\log (r+1))^{2}}\right)\right)^{2},
$$

which implies, via the inequality

$$
\frac{\log (r+1)}{\log r}=1+\frac{\log (1+1 / r)}{\log r}<1+\frac{1}{r \log r}
$$

that

$$
\begin{aligned}
x & <\frac{\log 2.2}{\log r}+1.374 \times 10^{6} n\left(\frac{\log (r+1)}{\log r}\right)(\log (r+1))^{2}\left(\log \left(\frac{0.3 m x}{(\log (r+1))^{2}}\right)\right)^{2} \\
& <\frac{\log 2.2}{\log r}+1.374 \times 10^{6} n\left(1+\frac{1}{r \log r}\right)(\log (r+1))^{2}\left(\log \left(\frac{0.3 m x}{3(\log (r+1))^{2}}\right)\right)^{2} \\
& <1.38 \times 10^{6} n\left(1+\frac{1}{r \log r}\right)(\log (r+1))^{2}\left(\log \left(\frac{0.3 m x}{(\log (r+1))^{2}}\right)\right)^{2} .
\end{aligned}
$$

Using the inequality (19), we know that $m<(n+1) x+2<(n+1)(x+1)$ (because $n \geq 2$ ), and substituting this in (33) we get that

$$
x<1.38 \times 10^{6} n\left(1+\frac{1}{r \log r}\right)(\log (r+1))^{2}\left(\log \left(\frac{0.3(n+1)(x+1)^{2}}{(\log r)^{2}}\right)\right)^{2} .
$$

We now turn our attention to the possibilities (i) and (ii). In case (i), there are positive integers $r_{0}, s_{0}$, which may be assumed to be coprime, such that $r_{0} b_{2}=s_{0} b_{1}$. So, we get $r_{0} m=s_{0}$ and since $r_{0}, s_{0}$ are coprime, we take $r_{0}=1, s_{0}=m$, and we get

$$
m=s_{0}<5.61 A_{1}(\mathcal{D} \log \mathcal{D})^{1 / 3}<5.61 \times 8.296 \times(2 \log 2)^{1 / 3} \log (r+1)
$$




$$
\begin{aligned}
& <5.61 \times 8.296 \times(2 \log 2)^{1 / 3} \log (r+1) \\
& <52 \log (r+1)
\end{aligned}
$$

Since $m>(n-1) x+1>x$, this situation gives

$$
x<52 \log (r+1) .
$$

This was in situation (i). In situation (ii), we have integers $r_{1}, s_{1}, t_{1}, t_{2}$ with $r_{1} s_{1} \neq 0$ and

$$
\left(t_{1} b_{1}+r_{1} b_{3}\right) s_{1}=r_{1} b_{2} t_{2}, \quad \operatorname{gcd}\left(r_{1}, t_{1}\right)=\operatorname{gcd}\left(s_{1}, t_{2}\right)=1
$$

Thus, for us, we have

$$
\left(t_{1}+r_{1} x\right) s_{1}=r_{1} m t_{2}, \quad \operatorname{gcd}\left(r_{1}, t_{1}\right)=\operatorname{gcd}\left(s_{1}, t_{2}\right)=1 .
$$

Reducing the above equation modulo $r_{1}$ we get $t_{1} s_{1} \equiv 0\left(\bmod r_{1}\right)$ and since $\operatorname{gcd}\left(t_{1}, r_{1}\right)=1$, we get that $r_{1} \mid s_{1}$. So, we put $s_{1}=r_{1} s_{1}^{\prime}$ and simplify both sides of the above equation by $r_{1}$ to get

$$
\left(t_{1}+r_{1} x\right) s_{1}^{\prime}=m t_{2}
$$

Consequently, for us $\delta=\operatorname{gcd}\left(r_{1}, s_{1}\right)=r_{1}$. Hence,

$$
\begin{aligned}
\left|t_{1} s_{1}^{\prime}\right| & <5.61 A_{1}(\mathcal{D} \log \mathcal{D})^{1 / 3}<5.61 \times 8.296 \log (r+1) \times(2 \log 2)^{1 / 3}<52 \log (r+1), \\
\left|t_{2}\right| & <5.61 A_{2}(\mathcal{D} \log \mathcal{D})^{1 / 3}<5.61 \times 6.296(2 \log 2)^{1 / 3} \log (r+1)<40 \log (r+1), \\
\left|r_{1} s_{1}^{\prime}\right| & <5.61 A_{3}(\mathcal{D} \log \mathcal{D})^{1 / 3}<5.61 \times 8.296(2 \log 2)^{1 / 3} n \log (r+1)<52 n \log (r+1) .
\end{aligned}
$$

Assume first that $t_{2}=0$. Then

$$
x=\left|t_{1}\right| /\left|r_{1}\right| \leq\left|t_{1}\right|<52 \log (r+1),
$$

which is the same as (35). Assume next that $t_{2} \neq 0$. We return to inequality (27) and multiply both sides by $t_{2}$ and get

$$
\left|m t_{2} \log \gamma_{2}-t_{2} \log \gamma_{1}-x t_{2} \log \gamma_{3}\right|<\frac{2.2\left|t_{2}\right|}{r^{x}}
$$

We substitute $m t_{2}$ by $t_{1} s_{1}^{\prime}+\left(r_{1} s_{1}^{\prime}\right) x$ inside the left-hand side above and then the left-hand side above becomes

$$
\left|\log \left(\frac{\gamma_{2}^{t_{1} s_{1}^{\prime}}}{\gamma_{1}^{t_{2}}}\right)+x \log \left(\frac{\gamma_{2}^{r_{1} s_{1}^{\prime}}}{\gamma_{3}^{t_{2}}}\right)\right|<\frac{2.2\left|t_{2}\right|}{r^{x}} .
$$


Inequality (37) is of the form

$$
\left|\Gamma_{1}\right|<\frac{2.2\left|t_{2}\right|}{r^{x}}, \quad \text { where } \quad \Gamma_{1}:=\log \eta_{1}+x \log \eta_{2}
$$

and

$$
\eta_{1}:=\frac{\gamma_{2}^{t_{1} s_{1}^{\prime}}}{\gamma_{1}^{t_{2}}}, \quad \eta_{2}:=\frac{\gamma_{2}^{r_{1} s_{1}^{\prime}}}{\gamma_{3}^{t_{2}}}
$$

We check that $\eta_{1}$ and $\eta_{2}$ are multiplicatively independent. If not, there are integers $i, j$ not both zero such that $\eta_{1}^{i} \eta_{2}^{j}=1$. This gives

$$
\left(\frac{\gamma_{2}^{t_{1} s_{1}^{\prime}}}{\gamma_{1}^{t_{2}}}\right)^{i}\left(\frac{\gamma_{2}^{r_{1} s_{1}^{\prime}}}{\gamma_{3}^{t_{2}}}\right)^{j}=1
$$

If $i \neq 0$, this gives a multiplicative dependence among $\gamma_{1}, \gamma_{2}, \gamma_{3}$ with the exponent of $\gamma_{1}$ being the nonzero integer $-t_{2} i$, a contradiction with the main result of Sect. 3.4. Thus, $i=0$, so $j \neq 0$, and we get again a multiplicative relation among $\gamma_{2}, \gamma_{3}$ (the exponent of $\gamma_{3}$ being the nonzero integer $-t_{2} j$ ), which is the same contradiction. Thus, indeed $\eta_{1}$ and $\eta_{2}$ are multiplicatively independent and they are also positive. So, we are in position to apply Theorem 3 to the left-hand side of inequality (38). We compute $\log B_{i}$ for these choices. We have, by the properties (15),

$$
\begin{aligned}
h\left(\eta_{1}\right) \leq & \left|t_{1} s_{1}^{\prime}\right| h\left(\gamma_{2}\right)+\left|t_{2}\right| h\left(\gamma_{1}\right) \leq(52 \log (r+1))(1 / 2 \log (r+1)) \\
& +(40 \log (r+1)) \log (r+1) \\
= & 66(\log (r+1))^{2} \\
h\left(\eta_{2}\right) \leq & \left|r_{1} s_{1}^{\prime}\right| h\left(\gamma_{2}\right)+\left|t_{2}\right| h\left(\gamma_{3}\right) \leq 52 n \log (r+1)(1 / 2 \log (r+1)) \\
& +(40 \log (r+1))(n \log (r+1)) \\
= & 66 n(\log (r+1))^{2} .
\end{aligned}
$$

Since $\left|\log \gamma_{i}\right| / 2 \leq h\left(\gamma_{i}\right)$ holds for $i=1,2,3$, it follows, by the absolute value inequality, that the same inequalities are satisfied by the numbers $\left|\log \eta_{i}\right| / 2$ for $i=1,2$. Thus, since $\mathcal{D}=2$, we can take

$$
\log B_{1}:=66(\log (r+1))^{2}, \quad \log B_{2}:=66 n(\log (r+1))^{2} .
$$

We bound

$$
\frac{1}{2 \log B_{2}}+\frac{x}{2 \log B_{1}}=\frac{1}{132(\log (r+1))^{2}}\left(\frac{1}{n}+x\right)<\frac{x+1}{132(\log (r+1))^{2}} .
$$

Hence, we take

$$
b^{\prime}:=\frac{x+1}{132(\log (r+1))^{2}}
$$


Now Theorem 3 gives

$$
\begin{aligned}
\log |\Lambda|> & -24.34 \times 2^{4}\left(\max \left\{\log b^{\prime}+0.14,10.5\right\}\right)^{2} \times\left(66(\log (r+1))^{2}\right) \\
& \times\left(66 n(\log (r+1))^{2}\right) \\
> & -1.627 \times 10^{6} n(\log (r+1))^{4} M^{2},
\end{aligned}
$$

where $M:=\max \left\{\log b^{\prime}+0.4,10.5\right\}$. In case $M=10.5$, we get

$$
b^{\prime}<e^{10.5-0.4}<24400
$$

which gives

$$
x+1<24400 \times 132(\log (r+1))^{2}<3.3 \times 10^{6}(\log (r+1))^{2} .
$$

Finally, suppose that

$$
M=\log b^{\prime}+0.4=\log \left(e^{0.4} b^{\prime}\right)<\log \left(1.5 b^{\prime}\right)=\log \left(\frac{x+1}{88(\log (r+1))^{2}}\right) .
$$

Comparing inequality (39) with inequality (38), we get

$$
x \log r-\log \left(2.2\left|t_{2}\right|\right)<1.627 \times 10^{6} n(\log (r+1))^{4}\left(\log \left(\frac{x+1}{88(\log (r+1))^{2}}\right)\right)^{2} .
$$

Since $2.2\left|t_{2}\right|<88 \log (r+1)$, we get that

$$
\begin{aligned}
x< & \frac{\log (88 \log (r+1))}{\log r} \\
& +1.627 \times 10^{6}\left(\frac{\log (r+1)}{\log r}\right)(\log (r+1))^{3}\left(\log \left(\frac{x+1}{88(\log (r+1))^{2}}\right)\right)^{2} .
\end{aligned}
$$

The first summand in the right-hand side is $<5$ for all $r \geq 3$. Using inequality (32), we get that

$$
x<1.63 \times 10^{6} n\left(1+\frac{1}{r \log r}\right)(\log (r+1))^{3}\left(\log \left(\frac{x+1}{88(\log (r+1))^{2}}\right)\right)^{2} .
$$

To summarise, either we are in the first situation of Theorem 2 and $\log \mathcal{B}=5$, in which case inequality (30) holds, or $\log \mathcal{B}>5$ in which case inequality (34) holds, or we are in the exceptional case (i) for which inequality (35) holds, which is contained in inequality (30), or we are in the exceptional situation (ii) in which case either inequality (40) holds, or inequality (63) holds. Since inequality (30) is contained in inequality (40), it follows, using the inequality $1 / 88<0.12$, that we proved the following result. 
Lemma 8 One of the following inequalities holds:

$$
\begin{aligned}
& x<3.3 \times 10^{6}(\log (r+1))^{2}, \\
& x<1.38 \times 10^{6} n\left(1+\frac{1}{r \log r}\right)(\log (r+1))^{2}\left(\log \left(\frac{0.3(n+1)(x+1)^{2}}{(\log (r+1))^{2}}\right)\right)^{2}, \\
& x<1.63 \times 10^{6} n\left(1+\frac{1}{r \log r}\right)(\log (r+1))^{3}\left(\log \left(\frac{0.12(x+1)}{(\log (r+1))^{2}}\right)\right)^{2} .
\end{aligned}
$$

\subsection{More inequalities in terms of $n$ and $x$}

We put

$$
\kappa:=n x+1-m
$$

Later we shall show that $\kappa$ is positive except possibly if $r=3$. In this section, assuming that it is positive, we show how it gives some lower bounds for $x$ in terms of $n$.

Lemma 9 The following holds :

(i) $\kappa \neq 1$,

(ii) If $\kappa=2$ and $n \geq 3$ then $x \geq r^{\max \{2, n-3\}}$,

(iii) If $\kappa \geq 3$, then $\kappa \geq n / 2$.

Proof (i). If $\kappa=1$, then $m=n x$. So Eq. (3) becomes

$$
U_{n}^{x}+U_{n+1}^{x}=U_{n x}
$$

If $p$ is prime dividing $U_{n}$ (which exists since $n>1$ ), then $p \mid U_{n}^{x}$ and $p\left|U_{n}\right| U_{n x}$, so from the above equation we get $p \mid U_{n+1}$, a contradiction since $\operatorname{gcd}\left(U_{n}, U_{n+1}\right)=$ $U_{\operatorname{gcd}(n, n+1)}=1$ by relation (12).

(ii) In this case $m=n x-1$ so Eq. (3) becomes

$$
U_{n}^{x}+U_{n+1}^{x}=U_{n x-1} .
$$

In particular, $U_{n x-1}-U_{n+1}^{x} \equiv 0\left(\bmod U_{n}^{2}\right)$. We study this congruence. In what follows for three algebraic integers $a, b, c$, we write $a \equiv b(\bmod c)$ if $(a-b) / c$ is an algebraic integer. Write

$$
U_{n}=\frac{\alpha^{n}-\beta^{n}}{\alpha-\beta} \quad \text { as } \quad \alpha^{n}=\beta^{n}+\sqrt{\Delta} U_{n} .
$$

Then

$$
\alpha^{n x}=\left(\beta^{n}+\sqrt{\Delta} U_{n}\right)^{x} \equiv \beta^{n x}+x \beta^{n(x-1)} \sqrt{\Delta} U_{n} \quad\left(\bmod \Delta U_{n}^{2}\right)
$$

Thus,

$$
U_{n x-1}=\frac{\alpha^{n x} \alpha^{-1}-\beta^{n x-1}}{\sqrt{\Delta}}
$$




$$
\begin{aligned}
& \equiv \frac{\left(\beta^{n x}+x \beta^{n(x-1)} \sqrt{\Delta} U_{n}\right) \alpha^{-1}-\beta^{n x-1}}{\alpha-\beta} \quad\left(\bmod \sqrt{\Delta} U_{n}^{2}\right) \\
& \equiv \frac{\beta^{n x} \alpha^{-1}-\beta^{n x} \beta^{-1}}{\alpha-\beta}+x \beta^{n(x-1)} \alpha^{-1} U_{n} \quad\left(\bmod U_{n}^{2}\right) \\
& \equiv \beta^{n x}+x \beta^{n(x-1)} \alpha^{-1} U_{n} \quad\left(\bmod U_{n}^{2}\right) .
\end{aligned}
$$

On the other hand,

$$
\begin{aligned}
U_{n+1}^{x} & =\left(\frac{\alpha^{n+1}-\beta^{n+1}}{\sqrt{\Delta}}\right)^{x} \\
& \equiv\left(\frac{\left(\beta^{n}+\sqrt{\Delta} U_{n}\right) \alpha-\beta^{n+1}}{\sqrt{\Delta}}\right)^{x} \quad\left(\bmod U_{n}^{2}\right) \\
& \equiv\left(\beta^{n}+U_{n} \alpha\right)^{x} \quad\left(\bmod U_{n}^{2}\right) \\
& \equiv \beta^{n x}+x \beta^{n(x-1)} \alpha U_{n} \quad\left(\bmod U_{n}^{2}\right) .
\end{aligned}
$$

Thus,

$$
\begin{aligned}
U_{n x-1}-U_{n+1}^{x} & \equiv\left(\beta^{n x}+x \beta^{n(x-1)} \alpha^{-1} U_{n}\right)-\left(\beta^{n x}+x \beta^{n(x-1)} \alpha U_{n}\right) \quad\left(\bmod U_{n}^{2}\right) \\
& \equiv x \beta^{n(x-1)}\left(\alpha^{-1}-\alpha\right) U_{n} \quad\left(\bmod U_{n}^{2}\right) \\
& \equiv-x \beta^{n(x-1} r U_{n} \quad\left(\bmod U_{n}^{2}\right) .
\end{aligned}
$$

In the last step above, we used the fact that $\alpha^{-1}-\alpha=-\beta-\alpha=-r$. Since the expression $U_{n x-1}-U_{n+1}^{x}$ is divisible by $U_{n}^{2}$, we get that $U_{n}^{2} \mid \beta^{n(x-1)} x r U_{n}$. Since $\beta$ is a unit, we get that $U_{n} \mid x r$. For $n=2$, this gives us nothing since $U_{2}=r$. For $n=3, U_{3}=r^{2}+1$ is coprime to $r$, so $U_{3} \mid x$, which gives $x \geq r^{2}+1>r^{2}$. For $n=4$, we have that $U_{4}=r\left(r^{2}+2\right)$ divides $r x$, so $r^{2}+2 \mid x$ giving $x \geq r^{2}+2>r^{2}$. Finally, for $n \geq 5$, we have that $U_{n}>\alpha^{n-2}>r^{n-2}$ and so $x \geq U_{n} / r \geq r^{n-3}$. This proves (ii).

(iii) We may assume that $n \geq 7$, otherwise the conclusion is trivial. Recall that $V_{n}=$ $\alpha^{n}+\beta^{n}$. Relations Eqs. (9) and (10) are

$$
U_{n+1}^{2}-U_{n} U_{n+2}=(-1)^{n} \quad \text { and } \quad V_{n}^{2}-\Delta U_{n}^{2}=4(-1)^{n} .
$$

In particular, $U_{n+1}^{2} \equiv(-1)^{n}\left(\bmod U_{n}\right)$ and $V_{n}^{2} \equiv 4(-1)^{n}\left(\bmod U_{n}\right)$. We also use the fact that $U_{-m}=(-1)^{m-1} U_{m}, V_{-m}=(-1)^{m} V_{m}$ and relation (11) which is

$$
2 U_{m+n}=U_{m} V_{n}+U_{n} V_{m} .
$$

Armed with these facts, writing $m=n x-(\kappa-1)$ and

$$
U_{n}^{x}+U_{n+1}^{x}=U_{n x-(\kappa-1)},
$$

we multiply both sides of the above equation by 2 and write

$$
2 U_{n}^{x}+2 U_{n+1}^{x}=2 U_{n x-(\kappa-1)}=U_{n x} V_{-(\kappa-1)}+V_{n x} U_{-(\kappa-1)} .
$$


We square both sides of the above equation and reduce it modulo $U_{n}$ taking into account that $U_{n} \mid U_{n x}$ and $V_{n x}^{2} \equiv 4(-1)^{n x}\left(\bmod U_{n x}\right) \equiv 4(-1)^{n x}\left(\bmod U_{n}\right)$, and get

$$
\begin{aligned}
4(-1)^{n x} & \equiv 4\left(U_{n+1}^{2}\right)^{x} \quad\left(\bmod U_{n}\right) \equiv\left(U_{n x} V_{-(\kappa-1)}+V_{n x} U_{-(\kappa-1)}\right)^{2} \quad\left(\bmod U_{n}\right) \\
& \equiv V_{n x}^{2} U_{-(\kappa-1)}^{2} \quad\left(\bmod U_{n}\right) \equiv V_{n x}^{2} U_{\kappa-1}^{2} \quad\left(\bmod U_{n}\right) \equiv 4(-1)^{n x} U_{\kappa-1}^{2} \quad\left(\bmod U_{n}\right)
\end{aligned}
$$

Thus, $U_{n} \mid 4\left(U_{\kappa-1}^{2}-1\right)$. The right-hand side is nonzero since $\kappa>2$. If $\kappa-1$ is odd, then

$$
4\left(U_{\kappa-1}^{2}-1\right)=4 U_{\kappa-2} U_{\kappa}
$$

by relation (9) (with $n+1:=\kappa-1$ ). Since $n \geq 7$ and $\mathbf{U}$ is not the Fibonacci sequence, it follows that $U_{n}$ has a primitive divisor $p$, which must divide one of $U_{\kappa-2}$ or $U_{\kappa}$. Thus, $z(p)=n$ divides one of $\kappa-2$ or $\kappa$, so we get $\kappa \geq n$, which is a better conclusion than the one desired. If $\kappa-1$ is even and $U_{n} \mid 2\left(U_{\kappa-1}^{2}-1\right)$, we then get

$$
\alpha^{n-2}<U_{n}<2 U_{\kappa-1}^{2}<r\left(\alpha^{\kappa-1}\right)^{2}<\alpha^{2 \kappa-1},
$$

so $2 \kappa-2 \geq n-2$, therefore $\kappa \geq n / 2$. The above argument was based on the fact that $r \geq 2$. In particular, if $r \geq 4$, then the same argument gives again that

$$
\alpha^{n-2}<U_{n}<4 U_{\kappa-1}^{2}<r\left(\alpha^{\kappa-1}\right)^{2}<\alpha^{2 \kappa-1},
$$

so $\kappa \geq n / 2$. So, the only case when the above arguments fail are when $r=3$ and $4 \mid U_{n}$. It then follows that $n$ is even (in fact, $n$ is a multiple of 6 , but we shall not need that), so $r\left|U_{n}\right| 4\left(U_{\kappa-1}^{2}-1\right)$. But $U_{\kappa-1}$ is a multiple of $r$ (since $\kappa-1$ is even), so $U_{\kappa-1}^{2}-1$ is coprime to $r$. Thus, $r \mid 4$, which is false. This finishes the proof of (iii).

Corollary 4 If $\kappa>0$, then $x>n / 2$.

Proof By Lemma 9, if $\kappa=2$, then $x \geq r^{\min \{2, n-3\}} \geq 3^{\min \{2, n-3\}}>n / 2$ for any $n \geq 2$. If $\kappa \geq 3$, then

$$
1+n x-m=\kappa \geq n / 2,
$$

which leads to $1+n x-n / 2 \geq m$. Comparing this with the lower bound $m>(n-1) x+1$ given by inequality (19), we get $x>n / 2$.

\subsection{Another inequality among $r, n, m, x$}

In this section, we return to inequality (27) and rewrite it in order to deduce a good approximation of $\log r$ by a rational number whose denominator is a multiple of $r^{2}$. Let's get to work. We need approximations of $\log \alpha$ and $\log \sqrt{r^{2}+4}$ in terms of $\log r$.

Lemma 10 For $r \geq 3$, we have the following approximations:

$$
\log \sqrt{r^{2}+4}=\log r+\frac{2}{r^{2}}+\zeta, \quad|\zeta|<\frac{5.81}{r^{4}} ;
$$




$$
\log \alpha=\log r+\frac{1}{r^{2}}+\zeta^{\prime}, \quad\left|\zeta^{\prime}\right|<\frac{3.64}{r^{4}} .
$$

Proof We have

$$
\log \sqrt{r^{2}+4}=\frac{1}{2} \log \left(r^{2}+4\right)=\log r+\frac{1}{2} \log \left(1+\frac{4}{r^{2}}\right) .
$$

With $z:=4 / r^{2}$, we have $|z| \leq 4 / 9$ and

$$
\log (1+z)=z+\zeta_{1}, \quad\left|\zeta_{1}\right| \leq \sum_{k \geq 2} \frac{z^{k}}{k}=\frac{z^{2}}{2}\left(1+(2 / 3) z+(2 / 4) z^{2}+\cdots\right)
$$

The expression in parenthesis above is smaller than

$$
c_{1}:=1+(2 / 3)(4 / 9)+(2 / 4)(4 / 9)^{2}+\cdots=(-4 / 9-\log (1-4 / 9)) \times 2 \times(9 / 4)^{2} .
$$

Hence,

$$
\begin{aligned}
\log \sqrt{r^{2}+4} & =\log r+\frac{2}{r^{2}}+\frac{\zeta_{1}}{2}:=\log r+\frac{2}{r^{2}}+\zeta, \quad|\zeta|=\frac{\left|\zeta_{1}\right|}{2} \\
& <\left(\frac{c_{1}}{4}\right) z^{2}=\frac{4 c_{1}}{r^{4}}<\frac{5.81}{r^{4}} .
\end{aligned}
$$

For $\alpha$, we write

$$
\begin{aligned}
\log \alpha & =\log r+\log \left(\frac{1}{2}+\sqrt{\frac{1}{4}+\frac{1}{r^{2}}}\right)=\log r+\log \left(1+z_{1}\right), \\
z_{1} & :=\sqrt{\frac{1}{4}+\frac{z}{4}}-\frac{1}{2} .
\end{aligned}
$$

Note that $\left|z_{1}\right| \leq 1 / r^{2}$. Thus,

$$
\log \left(1+z_{1}\right)=z_{1}+\zeta_{2}, \quad\left|\zeta_{2}\right| \leq \frac{\left|z_{1}\right|^{2}}{2}\left(1+(2 / 3)\left|z_{1}\right|+\cdots\right) \leq \frac{c_{2}}{2 r^{4}}
$$

where by the previous arguments,

$$
c_{2}=\left(-\lambda_{0}-\log \left(1-\lambda_{0}\right)\right) \times 2 \times \lambda_{0}^{-2}, \quad \text { with } \quad \lambda_{0}:=\sqrt{\frac{1}{4}+\frac{1}{9}}-\frac{1}{2} .
$$

It remains to expand $z_{1}$. For this, we have

$$
z_{1}=\frac{1}{2}(\sqrt{1+z}-1)=\frac{1}{2}\left(\frac{z}{2}+\zeta_{3}\right), \quad\left|\zeta_{3}\right| \leq \sum_{k \geq 2}\left|\left(\begin{array}{c}
k \\
1 / 2
\end{array}\right)\right| z^{k}
$$


Since $\left|\left(\begin{array}{c}k \\ 1 / 2\end{array}\right)\right| \leq 1 / 4$ for all $k \geq 1$, it follows that

$$
\left|\zeta_{3}\right| \leq \frac{1}{4} \sum_{k \geq 2} z^{k}=\frac{z^{2}}{4(1-z)} \leq \frac{36}{5 r^{4}}
$$

Hence,

$$
\begin{gathered}
\log \alpha=\log r+\frac{z}{4}+\left(\zeta_{2}+\frac{\zeta_{3}}{2}\right)=: \log r+\frac{1}{r^{2}}+\zeta^{\prime}, \\
\left|\zeta^{\prime}\right|<\left(\frac{c_{2}}{2}+\frac{36}{10}\right) \frac{1}{r^{2}}<\frac{3.64}{r^{4}}
\end{gathered}
$$

The following estimate is the main result of this section.

Lemma 11 If $r \geq 4$, then $\kappa>0$. Furthermore,

$$
x>\frac{\kappa r^{2} \log r+1}{1+5 / r} .
$$

Proof We shall use the approximations given in Lemma 10 but we also need an approximation of $\log U_{n+1}$. We have

$$
\log U_{n+1}=\log \left(\frac{\alpha^{n+1}}{\alpha-\beta}\left(1-\left(\frac{\beta}{\alpha}\right)^{n+1}\right)\right)=(n+1) \log \alpha-\log \left(\sqrt{r^{2}+4}\right)+\zeta^{\prime \prime}
$$

where

$$
\zeta^{\prime \prime}=\log \left(1-\left(\frac{\beta}{\alpha}\right)^{n+1}\right)
$$

Since $\beta=-\alpha^{-1}$, it follows that $|\beta / \alpha|=1 / \alpha^{2}$. Thus,

$$
\left|\zeta^{\prime \prime}\right| \leq \frac{1}{\alpha^{2 n+2}}\left(1+\sum_{k \geq 1} \frac{1}{k\left(\alpha^{2 n+2}\right)^{k}}\right) \leq \frac{1}{\alpha^{2 n+2}}\left(1+\frac{1}{2\left(1-1 / \alpha^{2 n+2}\right)}\right)<\frac{1.51}{\alpha^{2 n+2}},
$$

where for the last inequality we used the fact that $\alpha>r \geq 3$ and $n \geq 2$. Inserting estimates (46) and (47) together with Eq. (49) into inequality (27), we get

$$
\begin{aligned}
|\Gamma| & =\left|m \log \alpha-\log \left(\sqrt{r^{2}+4}\right)-x\left((n+1) \log \alpha-\log \left(\sqrt{r^{2}+4}\right)+\zeta^{\prime \prime}\right)\right| \\
& =\left|(m-x(n+1)) \log \alpha+(x-1) \log \left(\sqrt{r^{2}+4}\right)-x \zeta^{\prime \prime}\right| \\
& =\left|(m-x(n+1))\left(\log r+\frac{1}{r^{2}}+\zeta^{\prime}\right)+(x-1)\left(\log r+\frac{2}{r^{2}}+\zeta\right)-x \zeta^{\prime \prime}\right|
\end{aligned}
$$




$$
\begin{aligned}
= & \mid(m-n x-1) \log r+\frac{(m-n x-1)+(x-1)}{r^{2}} \\
& +(m-x(n+1)) \zeta^{\prime}+(x-1) \zeta+x \zeta^{\prime \prime} \mid .
\end{aligned}
$$

We recognise the coefficient of $\log r$ as the number we denoted $-\kappa$ in (45). Using inequality (27), we get

$$
\left|-\kappa \log r+\frac{-\kappa+(x-1)}{r^{2}}\right|<\frac{2.2}{r^{x}}+|m-x(n+1)|\left|\zeta^{\prime}\right|+|x-1||\zeta|+x\left|\zeta^{\prime \prime}\right| .
$$

Inequality (19) shows that $m-x(n+1) \in[-2 x+2,2]$. In particular, $|m-x(n+1)| \leq$ $2(x-1)$. We thus get, by estimates (46) and (47) together with Eq. (49), that

$$
\left|-\kappa \log r+\frac{-\kappa+(x-1)}{r^{2}}\right|<\frac{4}{r^{x}}+\frac{7.28(x-1)}{r^{4}}+\frac{5.81(x-1)}{r^{4}}+\frac{1.51 x}{r^{6}} .
$$

Since $x \geq 3$ and $r \geq 3$, we get that the last term satisfies

$$
\frac{1.51 x}{r^{6}} \leq\left(\frac{1.51 \times(3 / 2)}{3^{2}}\right) \frac{(x-1)}{r^{4}}<\frac{0.26(x-1)}{r^{4}} .
$$

Hence,

$$
\left|-\kappa \log r+\frac{-\kappa+(x-1)}{r^{2}}\right|<\frac{2.2}{r^{x}}+\frac{13.35(x-1)}{r^{4}} .
$$

Assume that $\kappa \leq 0$. We then get that

$$
\frac{x-1}{r^{2}} \leq \frac{2.2}{r^{x}}+\frac{13.35(x-1)}{r^{4}}
$$

which implies that

$$
1 \leq \frac{13.35}{r^{2}}+\frac{2.2}{r^{x-2}(x-1)}
$$

If $r \geq 5$, the right-hand side is

$$
\leq \frac{13.35}{25}+\frac{2.2}{5 \times 4}=\frac{13.54}{16}<1
$$

a contradiction. Similarly, if $r \geq 4$ and $x \geq 4$, then the right side is

$$
\leq \frac{13.25}{16}+\frac{2.2}{16 \times 3}<\frac{14}{16}<1
$$

Thus, if $r \geq 5$ or $r=4$ and $x \geq 4$, then $\kappa>0$ and now Lemma 9 applies. We will show at the end of this proof that $\kappa>0$ for $(r, x)=(4,3)$ as well. Multiplying both sides of 
estimate (51) by $r^{2}$, we get

$$
\left|-\kappa\left(r^{2} \log r+1\right)+(x-1)\right|<(x-1)\left(\frac{13.35}{r^{2}}+\frac{2.2}{r^{x-2}(x-1)}\right) .
$$

Hence,

$$
\kappa\left(r^{2} \log r+1\right)<(x-1)\left(1+\left(\frac{13.35}{r^{2}}+\frac{2.2}{r^{x-2}(x-1)}\right)\right) \leq(x-1)\left(1+\frac{5}{r}\right),
$$

which gives estimate (48). It remains to treat the case $(r, x)=(4,3)$. By inequality (19), we have $m<3(n+1)+1=3 n+4$ so $\kappa=3 n+1-m \geq-2$. So, the only instances in which $\kappa \leq 0$ is possible are when $m=3 n+3, m=3 n+2,3 n+1$. Well, let us show that this is not possible by proving that

$$
U_{n}^{3}+U_{n+1}^{3}<U_{3 n+1}
$$

Using the Binet formula (4), this is implied by

$$
\alpha^{-1}\left(1+\frac{1}{\alpha^{6 n}}\right)^{3}+\alpha^{2}\left(1+\frac{1}{\alpha^{6 n+6}}\right)^{3}<\Delta\left(1-\frac{1}{\alpha^{6 n+3}}\right)
$$

with $\alpha=2+\sqrt{5}$ and $\Delta=20$. The function of $n$ in the left is decreasing and the function with $n$ in the right is increasing, and the inequality holds at $n=1$ (the left-hand side there is $<18.5$ and the right side is $>19.5$ ), so it holds for all $n \geq 1$. Thus, $\kappa \geq 2$ for $r=4$ as well.

\subsection{The case $n \leq 100$}

We first seek bounds on $r$. Having the bounds in $r$ and $n$, we get bounds on $x$ using Lemma 8. Finally, for a fixed $r$ we use Baker-Davenport on estimate Eq. (27) to lower $x$. The hope is that in all cases $x \leq 100$, a case which has already been treated.

We prove the following result.

Lemma 12 When $n \leq 100$, we have $r \leq 1.5 \times 10^{6}$.

Proof We assume $r>10^{6}$. Then $x>\kappa r^{2} \log r / 1.01 \geq n r^{2} \log r / 2.02$ by Lemmas 9 and 11. We go through the three possibilities of Lemma 8. In case (i), we get

$$
r^{2} \log r \leq 1.01 \times 3.3 \times 10^{6}(\log (r+1))^{2},
$$

which gives $r<5500$, a contradiction. Assume we are in case (ii). Then

$$
x+1<1.38 \times 10^{6} \times 1.001(\log (r+1))^{2} \log \left(\frac{0.3(n+1)(x+1)^{2}}{(\log (r+1))^{2}}\right) .
$$


The factor 1.001 is an upper bound on the factor $1+1 /(r \log r)$ which is valid since $r$ is large. Now

$$
x+1>x>\frac{n r^{2} \log r}{2.02}>n r^{2} \log (r+1)\left(\frac{\log r}{2.02 \log (r+1)}\right)>\frac{n r^{2} \log (r+1)}{2.03},
$$

where the last inequality holds since $r>10^{6}$. Put $y:=(x+1) /(n(\log (r+1))$. Then the above inequality is $y>r^{2} / 2$.03. Inequality (52) can be rewritten in terms of $y$ as

$$
\begin{aligned}
y & <1.38 \times 10^{6} \log (r+1) \times 1.001\left(\log \left(0.3 n^{2}(n+1) y^{2}\right)\right)^{2} \\
& <1.39 \times 10^{6} \log (r+1)(2 \log y+\log (30,030))^{2} .
\end{aligned}
$$

We look at the function

$$
f(y):=\frac{y}{(2 \log y+\log (30,030))^{2}} .
$$

Its derivative is

$$
\frac{2 \log y+\log (30,030)-4}{(2 \log y+\log (30,030))^{3}}>0
$$

so our function $f(y)$ is increasing. Since $f(y)<1.39 \times 10^{6} \log (r+1)$, and $y>r^{2} / 2.03$, it follows that $f\left(r^{2} / 2.03\right)<1.39 \times 10^{6} \log (r+1)$. This gives

$$
r^{2}<1.39 \times 2.03 \times 10^{6}(\log (r+1))\left(2 \log \left(r^{2} / 2.03\right)+\log (30,030)\right)^{2},
$$

which gives $r<370000$, a contradiction. Assume we are in case (iii). We use the same substitution $y:=(x+1) /(n(\log (r+1))$. We then get

$$
\begin{aligned}
y< & 1.64 \times 10^{6}(\log (r+1))^{2}\left(\log \left(0.12 n^{2} y^{2}\right)\right)^{2} \leq 1.64 \\
& \times 10^{6}(\log (r+1))^{2}(2 \log y+\log (12))^{2} .
\end{aligned}
$$

The function $g(y):=y /(2 \log y+\log (12))^{2}$ is also increasing, so we deduce that

$$
r^{2}<2.03 \times 1.64 \times 10^{6}(\log (r+1))^{2}\left(2 \log \left(r^{2} / 2.03\right)+\log (12)\right)^{2},
$$

and this gives $r<1.5 \times 10^{6}$.

Having bounds on $n$ and $r$, inequalities (i), (ii) and (iii) from Lemma 8 become

$$
\begin{aligned}
x & <3.3 \times 10^{6}\left(\log \left(1.5 \times 10^{6}\right)\right)^{2}<7 \times 10^{8}, \\
x & <1.39 \times 10^{6} \times 100\left(\log \left(1.5 \times 10^{6}\right)\right)^{2}\left(\log \left(0.3 \times 101(x+1)^{2}\right)\right)^{2} \\
& <3.7 \times 10^{10} \log (30.3(x+1))^{2},
\end{aligned}
$$




$$
\begin{aligned}
x & <1.64 \times 10^{6} \times 100 \times\left(\log \left(1.5 \times 10^{6}\right)\right)^{3}\left(\log (0.12(x+1))^{2}\right. \\
& <6.5 \times 10^{11} \log (0.12(x+1))^{2} .
\end{aligned}
$$

Any one of these inequalities implies that $x<3 \times 10^{15}$. Now we do Baker-Davenport on estimates (27) for $n \in[2,100], r \in[3,1,500,000]$, and $x<3 \times 10^{15}$. This also gives $m<3 \times 10^{17}$ via inequality (19). We return to inequality (27) and rewrite it as follows:

$$
\left|x \frac{\log U_{n+1}}{\log \alpha}-m+\frac{\log \left(\sqrt{r^{2}+4}\right)}{\log \alpha}\right|<\frac{2.2}{r^{x} \log \alpha} .
$$

Then, we apply Lemma 5 on Eq. (53) with the data:

$$
M:=3 \times 10^{17}, \quad \tau:=\frac{\log U_{n+1}}{\log \alpha}, \quad \mu:=\frac{\log \left(\sqrt{r^{2}+4}\right)}{\log \alpha}, \quad A:=\frac{2.2}{\log \alpha}, \quad \text { and } \quad B:=r .
$$

A computer search in Mathematica reveals that $x \leq 81$, which is a contradiction. This computation lasted 16 hours on a cluster of four 16 GB RAM computers.

\subsection{The case $n>100$}

Estimate (27) together with estimate (49) give

$$
|\Gamma|=\mid m \log \alpha-\log \left(\sqrt{r^{2}+4}-x\left((n+1) \log \alpha-\log \left(\sqrt{r^{2}+4}+\zeta^{\prime \prime}\right) \mid<\frac{2.2}{r^{x}},\right.\right.
$$

which implies, via estimate (50), that

$$
\left|(m-x(n+1)) \log \alpha+(x-1) \log \left(\sqrt{r^{2}+4}\right)\right|<\frac{2.2}{r^{x}}+\frac{1.51 x}{\alpha^{2 n+2}} .
$$

If $r \geq 4$ then $\kappa>0$, so by Lemma 9 , we have $\kappa \geq 2$. If $\kappa \geq 3$, then

$$
x \geq \frac{\kappa\left(r^{2} \log r+1\right)}{1+5 / r}>\frac{n 4^{2} \log 4}{4.5}>2 n+2 .
$$

The same conclusion holds for $\kappa=2$ since then $x \geq r^{\min \{2, n-3\}} \geq 4^{\min \{2, n-3\}} \geq 2 n+2$ for all $n \geq 2$. We thus get

$$
\left|(m-x(n+1)) \log \alpha+(x-1) \log \left(\sqrt{r^{2}+4}\right)\right|<\frac{4}{r^{2 n+2}}+\frac{1.51 x}{\alpha^{2 n+2}}<\frac{1.51 x+2.2}{\alpha^{2 n+2}} .
$$

We prove the following lemma.

Lemma 13 For $n>100$, we have $r^{n-2}>x$.

Note that Lemmas 9 and 13 show that the case $\kappa=2$ cannot occur for $r \geq 4$ provided that $n>100$. 
Proof Assume $x \geq r^{n-2}$. We use the bounds given by Lemma 8 on $x$. In case (i), we get

$$
r^{99} \leq r^{n-2} \leq 3.3 \times 10^{6}(\log (r+1))^{2} .
$$

The function $y \mapsto y^{99} /(\log (y+1))^{2}$ is increasing for all $y \geq 3$ as one can check by computing its derivative. Thus, if the above inequality holds for $r$, it should hold $r=3$ as well, which is false. In case (ii), we have

$$
x<1.38 \times 10^{6} n\left(1+\frac{1}{r \log r}\right)(\log (r+1))^{2}\left(\log \left(\frac{0.3(n+1)(x+1)^{2}}{(\log (r+1))^{2}}\right)\right)^{2} .
$$

The expression $1+1 /(r \log r)$ is smaller than 1.304 at $r=3$. Since $1.38 \times 1.304<1.8$, we get

$$
x<1.8 \times 10^{6}(\log (r+1))^{2}\left(\log \left(\frac{0.3(n+1)(x+1)^{2}}{(\log (r+1))^{2}}\right)\right)^{2} .
$$

If $x \leq n$, then we get $r^{n-2} \leq n$ for $r \geq 3$ and $n \geq 101$, which is false. Thus, $n<x$, so we may use $0.3(n+1)<n<x+1$ to get

$$
\begin{aligned}
x+1 & <1.8 \times 10^{6} n(\log (r+1))^{2}\left(\log \left((x+1)^{3}\right)\right)^{2} \\
& <1.8 \times 3^{2} \times 10^{6}(\log (r+1))^{2}(\log (x+1))^{2} \\
& <1.7 \times 10^{7}(\log (r+1))^{2}(\log (x+1))^{3} .
\end{aligned}
$$

The function $(x+1) /(\log (x+1))^{3}$ is increasing for $x+1>e^{3}$, which is the case for us since $x \geq r^{99} \geq 3^{99}$. Hence, the above inequality should hold for $x+1$ replaced by $r^{n-2}$, and that gives

$$
r^{n-2} \leq 1.7 \times 10^{7}(\log (r+1))^{2}\left(\log \left(r^{n-2}\right)\right)^{2}<1.7 \times 10^{7} n^{2}(\log (r+1))^{4} .
$$

Since $r^{n / 3} \geq 3^{n / 3}>n$ holds for $n>100$, we get that

$$
r^{n / 3-2}<1.7 \times 10^{7}(\log (r+1))^{4},
$$

so

$$
r^{95} \leq r^{n-6}<\left(1.7 \times 10^{7}(\log (r+1))^{2}\right)^{3}<5 \times 10^{21}(\log (r+1))^{12}
$$

The function $y \mapsto y^{95} /(\log (y+1))^{6}$ is increasing for $y \geq 3$, so the last inequality should hold also for $r$ replaced by 3 , which is false. A similar argument works if $x$ is in case (iii) of Lemma 8. We don't give further details.

From Lemma 13 we conclude that if $r \geq 4$, then inequality (55) leads to

$$
\left|(m-x(n+1)) \log \alpha+(x-1) \log \left(\sqrt{r^{2}+4}\right)\right|<\frac{1}{r^{n}} .
$$


We put

$$
\Gamma_{2}:=(x-1) \log \left(\sqrt{r^{2}+4}\right)-((n+1) x-m) \log \alpha .
$$

We apply Theorem 3 to find a lower bound on $\log \left|\Gamma_{2}\right|$ with the data:

$$
t:=2, \quad \gamma_{1}:=\sqrt{r^{2}+4}, \quad \gamma_{2}:=\alpha, \quad b_{1}:=x-1, \quad b_{2}:=m-(n+1) x .
$$

Since $\gamma_{1}, \gamma_{2} \in \mathbb{Q}(\alpha)$, we take again $\mathbb{K}:=\mathbb{Q}(\alpha)$ with degree $\mathcal{D}:=2$. The fact that $\gamma_{1}$ and $\gamma_{2}$ are multiplicatively independent has already been checked.

We take

$$
\max \left\{h\left(\gamma_{1}\right), \frac{\left|\log \gamma_{1}\right|}{2}, \frac{1}{2}\right\}=\frac{1}{2} \log \left(r^{2}+4\right)<\log (r+1):=\log B_{1},
$$

and

$$
\max \left\{h\left(\gamma_{2}\right), \frac{\left|\log \gamma_{2}\right|}{2}, \frac{1}{2}\right\}=\frac{1}{2} \log \alpha<\frac{1}{2} \log (r+1):=\log B_{2} .
$$

Thus,

$$
b^{\prime}:=\frac{\left|b_{1}\right|}{D \log B_{2}}+\frac{\left|b_{2}\right|}{D \log B_{1}}=\frac{x-1}{2 \log (r+1)}+\frac{|(n+1) x-m|}{\log (r+1)}<\frac{2.5 x}{\log (r+1)},
$$

where we used the fact that $m-(n+1) x \in[-2 x+2,2]$. By Theorem 3 , we get that

$$
\log \left|\Gamma_{2}\right|>-195\left(\max \left\{\log \left(\frac{2.5 x}{\log r}\right), 10.5\right\}\right)^{2}(\log (r+1))^{2} .
$$

We want an upper bound on $r$. So, assume $r \geq 10^{6}$. If $\log ((2.5 x) / \log (r+1))<10.5$, then

$$
x<\frac{e^{10.5} \log (r+1)}{2.5}<15,000 \log (r+1)
$$

Since $x>n r^{2} \log r /(2+10 / r) \geq 101 r^{2} \log r / 2.02=50 r^{2} \log r$, we get

$$
50 r^{2} \log r \leq x \leq 15,000(\log (r+1))
$$

so

$$
r^{2}<\left(\frac{15,000}{50}\right)\left(\frac{\log (r+1)}{\log r}\right)<300 \times 1.01=303
$$

so $r \leq 17$, a contradiction. 
Assume next that $\log (2.5 x / \log (r+1))>10.5$. We then get

$$
\log \left|\Gamma_{2}\right|>-195(\log (r+1))^{2}\left(\log \left(\frac{2.5 x}{\log (r+1)}\right)\right)^{2}
$$

Comparing the above inequality with estimate (56), we get

$$
n \log r<195(\log (r+1))^{2}\left(\log \left(\frac{2.5 x}{\log (r+1)}\right)\right)^{2}
$$

so

$$
\begin{aligned}
n & <195\left(\frac{\log (r+1)}{\log r}\right) \log (r+1)\left(\log \left(\frac{2.5 x}{\log (r+1)}\right)\right)^{2} \\
& <196 \log (r+1)\left(\log \left(\frac{2.5 x}{\log (r+1)}\right)\right)^{2}
\end{aligned}
$$

where we used the fact that $r \geq 10^{6}$ so $\log (r+1) / \log r<1.0001$.

We now use the bounds on $x$ given by Lemma 8 . In case (i), we have

$$
50 r^{2} \log r \leq x \leq 3.3 \times 10^{6}(\log (r+1))^{2},
$$

so

$$
r^{2}<\left(\frac{3.3 \times 10^{6}}{50}\right) \times\left(\frac{\log (r+1)}{\log r}\right) \log (r+1)<6.7 \times 10^{4} \log (r+1)
$$

so $r<670$, a contradiction. In case (ii), we have

$$
x+1<1.39 \times 10^{6} n(\log (r+1))^{2}\left(\log \left(\frac{0.3(n+1)(x+1)^{2}}{(\log (r+1))^{2}}\right)\right)^{2} .
$$

In case $(x+1) /(\log (r+1)) \leq n$, we have

$$
x+1<196(\log (r+1))^{2}\left(\frac{2.5 x}{\log (r+1)}\right)^{2} .
$$

So, putting $y:=x / \log (r+1)$, we get $y<196 \log (r+1) \log (2.5 y)^{2}$. Note that

$$
y=\frac{(x+1)}{\log (r+1)}>\frac{n r^{2} \log r}{2.01 \log (r+1)} \geq \frac{101 r^{2}}{2.01(\log (r+1) / \log r)}>\frac{101 r^{2}}{2.02}=50 r^{2} .
$$

In the above inequalities we used the fact that $r \geq 10^{6}$. The function $y \mapsto$ $y / \log (2.5 y)^{2}$ is increasing for $y>e^{2} / 2.5$, which is our case. Hence, the inequality $y<196 \log (r+1) \log (2.5 y)^{2}$ should hold with $y$ replaced by $50 r^{2}$, which yields 
$50 r^{2}<196 \log (r+1) \log \left(2.5 \times 50 r^{2}\right)^{2}$ and gives $r<50$, a contradiction. Thus, $n<(x+1) / \log (r+1)$. Since $0.3(n+1)<n$, we conclude that

$$
\begin{aligned}
x+1< & 1.39 \times 10^{6}(\log (r+1))^{2}\left(196 \log (r+1)\left(\log \left(\frac{2.5 x}{\log (r+1)}\right)\right)^{2}\right) \\
& \times\left(\log \left(\left(\frac{(x+1)}{\log (r+1)}\right)^{3}\right)\right)^{2} .
\end{aligned}
$$

Putting again $y:=(x+1) / \log (r+1)$, we get

$$
y<1.39 \times 10^{6} \times 196 \times(\log (r+1))^{2} \log (2.5 y)^{2}(3 \log y)^{2}<2.46 \times 10^{9}(\log (2.5 y))^{4} .
$$

The function $y \mapsto y /\left(\log (2.5 y)^{4}\right.$ is increasing for our range for $y>50 r^{2}>50 \times 10^{16}$, so we get that the above inequality should hold by replacing $y$ by $50 r^{2}$. Thus,

$$
50 r^{2}<2.46 \times 10^{9}(\log (r+1))^{2}\left(\log \left(2.5 \times 50 r^{2}\right)\right)^{4},
$$

so $r<2.6 \times 10^{8}$. A similar argument holds when $x$ is in case (iii). In that case, we may again suppose that $n<(x+1) / \log (r+1)$. We get

$$
\begin{aligned}
x+1< & 1.64 \times 10^{6}(\log (r+1))^{3}\left(196 \log (r+1)\left(\log \left(\frac{2.5 x}{\log (r+1)}\right)\right)^{2}\right) \\
& \times\left(\log \left(\frac{0.12(x+1)}{(\log (r+1))^{2}}\right)\right)^{2},
\end{aligned}
$$

so

$$
y<3.3 \times 10^{8}(\log (r+1))^{3}(\log (2.5 y))^{2}(\log (0.12 y))^{2} .
$$

Imposing that the above inequality holds for $y$ replaced by $50 r^{2}$, we get

$$
50 r^{2}<1.3 \times 10^{9}(\log (r+1))^{3}\left(\log \left(2.5 \times 50 r^{2}\right)\right)\left(\log \left(0.12 \times 50 r^{2}\right)\right)^{2},
$$

so $r<4.3 \times 10^{8}$.

To summarise, we have proved the following.

Lemma 14 If $r \geq 4$, then $r<4.3 \times 10^{8}$.

Having bounds for $r$ it is easy to find bounds for $x$. For example,

$$
n<195 \log (r+1)(\log (2.5 x))^{2}<195 \log \left(4.3 \times 10^{8}\right)(\log (2.5 x))^{2}<3900(\log (2.5 x))^{2} .
$$

Next, if $x$ is in case (i), then

$$
x<3.3 \times 10^{6}(\log (r+1))^{2}<3.3 \times 10^{6}\left(\log \left(4.3 \times 10^{8}\right)\right)^{2}<1.4 \times 10^{9} .
$$


If $x$ is in case (ii), then

$$
\begin{aligned}
x< & 1.39 \times 10^{6}\left(\log \left(4.3 \times 10^{8}\right)^{2}\left(3900(\log (2.5 x))^{2}\right)\right. \\
& \times\left(\log \left(0.3 \times 3900(\log (2.5 x))^{2}(x+1)^{2}\right)\right)^{2} \\
< & 2.2 \times 10^{12}(\log (2.5 x))^{2}\left(\log \left(1200(x+1)^{2} \log (2.5 x)\right)\right)^{2},
\end{aligned}
$$

which gives $x<5 \times 10^{19}$. Finally, if $x$ is as in case (iii), then

$$
\begin{aligned}
x & <1.64 \times 10^{6}\left(\log \left(4.3 \times 10^{8}\right)^{3} \times 3900(\log (2.5 x))^{2} \times(\log (0.12(x+1)))^{2}\right. \\
& <5.1 \times 10^{13}(\log (2.5 x))^{2}(\log (0.12(x+1)))^{2},
\end{aligned}
$$

so $x<3 \times 10^{20}$.

Thus, $r<4.3 \times 10^{8}$ and $x<3 \times 10^{20}$. Inequality (56) gives that

$$
\left|\frac{\log \sqrt{r^{2}+4}}{\log \alpha}-\frac{x(n+1)-1}{x-1}\right|<\frac{1}{r^{n}(x-1) \log \alpha}<\frac{1}{16(x-1)^{2}},
$$

where for the last inequality we used that $r^{n}=r^{2} r^{n-2} \geq 16 x$ by Lemma 13. In particular, the ratio $(x(n+1)-1) /(x-1)$ is a convergent of $\log \sqrt{r^{2}+4} / \log \alpha$. Since $x<3 \times 10^{20}<$ $F_{100}$, it follows that $(x(n+1)-1) /(x-1)=p_{k} / q_{k}$ for some $k \in[0,99]$. So, we apply Lemma 4 on Eq. (59) with the data:

$$
M:=3 \times 10^{20}, \quad \tau:=\frac{\log \sqrt{r^{2}+4}}{\log \alpha}, \quad u:=x(n+1)-1, \quad \text { and } \quad v:=x-1 .
$$

With the help of a computer search in Mathematica, we checked all these possibilities over all the values for $4 \leq r \leq 4.3 \times 10^{8}$ and found that $n \leq 46$, which is a contradiction. This computation lasted 6 hours on a cluster of four 16 GB RAM computers.

\subsection{The case $r=3$}

The case $r=3$ is special since there we don't know that $\kappa>0$ so some of the inequalities used for the case $r \geq 4$ do not apply. In the case $n \leq 100$, Lemma 8 gives

$$
x<3.3 \times 10^{6} \times(\log 4)^{2}<6.4 \times 10^{6},
$$

or

$$
\begin{aligned}
x & <1.38 \times 10^{8} \times 100\left(1+\frac{1}{3 \log 3}\right)(\log 4)^{2}\left(\log \left(\frac{0.3 \times 101(x+1)^{2}}{(\log 4)^{2}}\right)\right), \\
& <3.5 \times 10^{10}\left(\log \left(16(x+1)^{2}\right)\right)^{2}
\end{aligned}
$$

which gives $x<1.7 \times 10^{14}$, or 


$$
\begin{aligned}
x & <1.63 \times 10^{6} \times 100\left(1+\frac{1}{3 \log 3}\right)(\log 4)^{3}\left(\log \left(\frac{0.12(x+1)}{(\log 4)^{2}}\right)\right)^{2} \\
& <5.7 \times 10^{8}(0.07(x+1))^{2},
\end{aligned}
$$

which gives $x<3.3 \times 10^{11}$. Now we can do Baker-Davenport on estimate (27) and get better bounds on $x$. In case $n>100$, estimate (54) together with Lemma 13 hold and give

$$
\begin{aligned}
\left|(m-x(n+1)) \log \alpha+(x-1) \log \left(\sqrt{r^{2}+4}\right)\right| & <\frac{2.2}{r^{x}}+\frac{1.51 x}{\alpha^{2 n+2}} \\
& <\frac{2.2}{r^{x}}+\frac{1.51}{r^{n+2}}<\frac{1}{3^{\min \{n-1, x-1\}}} .
\end{aligned}
$$

We keep the notation $r$ and $\alpha$ although this section only applies to $r=3$ for which $\alpha=(3+\sqrt{13}) / 2$. Put $\ell:=\min \{n-1, x-1\}$. The lower bound estimate (58) still applies and gives

$$
\ell \log 3<-\log \left|\Gamma_{2}\right|<195\left(\max \left\{\log \left(\frac{2.5 x}{\log 4}\right), 10.5\right\}\right)^{2}(\log 4)^{2}
$$

When $\ell=n-1$, we get

$$
(n-1) \log 3<375\left(\max \left\{\log \left(\frac{2.5 x}{\log 4}\right), 10.5\right\}\right)^{2} .
$$

In case the maximum above is 10.5 we get $x<16,000$ and $n<40,000$. If the maximum above is not at 10.5 , we then get

$$
n<\frac{375}{\log 3}\left(\log \left(\frac{2.5 x}{\log 4}\right)\right)^{2}+1<350(\log (2 x))^{2} .
$$

Going via the possibilities (i), (ii), (iii), we get

$$
\begin{aligned}
x<3.3 & \times 10^{6}(\log 4)^{2}<7 \times 10^{6} ; \\
x<1.38 & \times 10^{6}\left(350(\log (2 x))^{2}\right)\left(1+\frac{1}{3 \log 3}\right)(\log 4)^{2} \\
& \times\left(\log \left(\frac{0.3 \times 351(\log 2 x)^{2}(x+1)^{2}}{(\log 4)^{2}}\right)\right)^{2} \\
< & \left.1.3 \times 10^{9}(\log 2 x)\right)^{2}\left(\log \left(55(x+1)^{2}\right)\right)^{2},
\end{aligned}
$$

which gives $x<2 \times 10^{16}$, or finally

$$
\begin{aligned}
x & <1.63 \times 10^{6}\left(350(\log (2 x))^{2}\right)\left(1+\frac{1}{3 \log 3}\right)(\log 4)^{3}\left(\log \left(\frac{0.12(x+1)}{(\log 4)^{2}}\right)\right)^{2} \\
& <2 \times 10^{9}(\log (2 x))^{2}(\log (0.07(x+1)))^{2},
\end{aligned}
$$


which gives $x<3.1 \times 10^{15}$. So, in all instances, $x<2 \times 10^{16}$, and now

$$
n<350(\log (2 x))^{2}<6 \times 10^{5} .
$$

Since Lemma 13 still applies, it follows that estimate (60) gives

$$
\left|\frac{\log \sqrt{r^{2}+4}}{\log \alpha}-\frac{x(n+1)-m}{x-1}\right|<\frac{1}{3^{n-1}(x-1)(\log 3)}<\frac{1}{3(x-1)^{2}},
$$

so again $(x(n+1)-m) /(x-1)=p_{k} / q_{k}$ is a convergent of $\log \sqrt{r^{2}+4} / \log \alpha$ with $x<2 \times 10^{16}<F_{80}$, so $k \in[0, \ldots, 16]$. So, everything works fine if $\ell=n-1$.

In case $\ell=x-1$, one gets

$$
(x-1) \log 3<375\left(\max \left\{\log \left(\frac{2.5 x}{\log 4}\right), 10.5\right\}\right)^{2},
$$

which gives $x<5 \times 10^{4}$. And one wonders how one should finish it off. We can expand another linear form in logarithms which is small, or we may recall the following main theorem from [2].

Theorem 5 Assume that $s \notin\{1,2,4\}$ is minimal such that $U_{m} \mid U_{n+1}^{s}-U_{n}^{s}$. Then $m<20,000 s^{2}$.

In our instance, since $U_{m}=U_{n}^{x}+U_{n+1}^{x}$, one checks that the minimal $s$ is exactly $2 x$. Thus, $m<80,000 x^{2}$, and since $m>(n-1) x$ by estimate (19), we get $n \leq 80,000 x<$ $4 \times 10^{9}$. Thus, $n<4 \times 10^{9}$ and $x<5 \times 10^{4}$. It is still a large range and we need to reduce it.

We consider the element

$$
y:=\frac{x}{\alpha^{2 n}}
$$

Lemma 13 together with the fact that $\alpha>r$ implies that

$$
y<\frac{1}{\alpha^{n}}
$$

where the last inequality holds for all $n>100$. Now, we write

$$
U_{n}^{x}=\frac{\alpha^{n x}}{\left(r^{2}+4\right)^{x / 2}}\left(1-\frac{(-1)^{n}}{\alpha^{2 n}}\right)^{x},
$$

and

$$
U_{n+1}^{x}=\frac{\alpha^{(n+1) x}}{\left(r^{2}+4\right)^{x / 2}}\left(1-\frac{(-1)^{n+1}}{\alpha^{2(n+1)}}\right)^{x}
$$


If $n$ is odd, then

$$
1<\left(1-\frac{(-1)^{n}}{\alpha^{2 n}}\right)^{x}=\left(1+\frac{1}{\alpha^{2 n}}\right)^{x}<e^{y}<1+2 y
$$

because $y$ is very small. On the other hand, if $n$ is even, then

$$
1>\left(1-\frac{(-1)^{n}}{\alpha^{2 n}}\right)^{x}=\exp \left(x \log \left(1-\frac{1}{\alpha^{2 n}}\right)\right)^{x}>e^{-2 y}>1-2 y,
$$

because $y$ is very small. Thus, the following inequalities hold in both cases,

$$
\left|U_{n}^{x}-\frac{\alpha^{n x}}{\left(r^{2}+4\right)^{x / 2}}\right|<\frac{2 y \alpha^{n x}}{\left(r^{2}+4\right)^{x / 2}},
$$

and

$$
\left|U_{n+1}^{x}-\frac{\alpha^{(n+1) x}}{\left(r^{2}+4\right)^{x / 2}}\right|<\frac{2 y \alpha^{(n+1) x}}{\left(r^{2}+4\right)^{x / 2}} .
$$

Now, we return to (3) and rewrite it as

$$
\begin{aligned}
\frac{\alpha^{m}-\beta^{m}}{\left(r^{2}+4\right)^{1 / 2}}= & U_{m}=U_{n}^{x}+U_{n+1}^{x} \\
= & \frac{\alpha^{n x}}{\left(r^{2}+4\right)^{x / 2}}+\frac{\alpha^{(n+1) x}}{\left(r^{2}+4\right)^{x / 2}} \\
& +\left(U_{n}^{2}-\frac{\alpha^{n x}}{\left(r^{2}+4\right)^{x / 2}}\right)+\left(U_{n+1}^{x}-\frac{\alpha^{(n+1) x}}{\left(r^{2}+4\right)^{x / 2}}\right),
\end{aligned}
$$

or

$$
\begin{aligned}
& \left|\frac{\alpha^{m}}{\left(r^{2}+4\right)^{1 / 2}}-\frac{\alpha^{n x}\left(1+\alpha^{x}\right)}{\left(r^{2}+4\right)^{x / 2}}\right| \\
& \quad=\left|\frac{\beta^{m}}{\left(r^{2}+4\right)^{1 / 2}}+\left(U_{n}^{x}-\frac{\alpha^{n x}}{\left(r^{2}+4\right)^{x / 2}}\right)+\left(U_{n+1}^{x}-\frac{\alpha^{(n+1) x}}{\left(r^{2}+4\right)^{x / 2}}\right)\right| \\
& \quad<\frac{1}{\alpha^{m}}+\left|U_{n}^{x}-\frac{\alpha^{n x}}{\left(r^{2}+4\right)^{x / 2}}\right|+\left|U_{n+1}^{x}-\frac{\alpha^{(n+1) x}}{\left(r^{2}+4\right)^{x / 2}}\right| \\
& \quad<\frac{1}{\alpha^{m}}+2 y\left(\frac{\alpha^{n x}\left(1+\alpha^{x}\right)}{\left(r^{2}+4\right)^{x / 2}}\right) .
\end{aligned}
$$

Multiplying both sides of the above inequality by $\alpha^{-(n+1) x}\left(r^{2}+4\right)^{x / 2}$, we obtain that 


$$
\begin{aligned}
& \left|\alpha^{m-(n+1) x}\left(r^{2}+4\right)^{(x-1) / 2}-\left(1+\alpha^{-x}\right)\right|<\frac{\left(r^{2}+4\right)^{x / 2}}{\alpha^{m+(n+1) x}}+2 y\left(1+\alpha^{-x}\right) \\
& \quad<\frac{1}{2 \alpha^{n}}+3 y<\frac{3}{\alpha^{n}}
\end{aligned}
$$

so we may divide both sides of it by $1+\alpha^{-x}$ and get

$$
\left|\alpha^{m-(n+1) x}\left(r^{2}+4\right)^{(x-1) / 2}\left(1+\alpha^{-x}\right)^{-1}-1\right|<\frac{3}{\alpha^{n}} .
$$

Since $n>100$, the left-hand side is small (say smaller than $1 / 2$ ), so we can pass to a logarithmic form and get

$$
\left|(m-(n+1) x) \log \alpha+(x-1) \log \left(\sqrt{r^{2}+4}\right)-\log \left(1+\alpha^{-x}\right)\right|<\frac{6}{\alpha^{n}}
$$

For us, the parameter $x$ is in [3,50000]. Given $x$, we have $m<2 \times 10^{14}$. So, this is a suitable inequality to apply Baker-Davenport to. To do so, we rewrite Eq. (63) as

$$
\left|((n+1) x-m) \frac{\log \alpha}{\log \left(\sqrt{r^{2}+4}\right)}-(x-1)+\frac{\log \left(1+\alpha^{-x}\right)}{\log \left(\sqrt{r^{2}+4}\right)}\right|<\frac{6}{\alpha^{n} \log \left(\sqrt{r^{2}+4}\right)} .
$$

Then, we apply Lemma 5 on Eq. (64) with the data:

$$
\begin{aligned}
& M:=2 \times 10^{14}, \quad \tau:=\frac{\log \alpha}{\log \left(\sqrt{r^{2}+4}\right)}, \quad \mu:=\frac{\log \left(1+\alpha^{-x}\right)}{\log \left(\sqrt{r^{2}+4}\right)}, \\
& A:=\frac{6}{\log \left(\sqrt{r^{2}+4}\right)}, B:=\alpha .
\end{aligned}
$$

A computer search in Mathematica reveals that $n \leq 94$, which is the final contradiction. This computations lasted a few hours on a cluster four 16 GB RAM computers.

Acknowledgements This paper was written when both authors visited the Max Planck Institute for Mathematics Bonn, in 2019. They thank this institution for the hospitality and the fruitful working environment.

Open Access This article is licensed under a Creative Commons Attribution 4.0 International License, which permits use, sharing, adaptation, distribution and reproduction in any medium or format, as long as you give appropriate credit to the original author(s) and the source, provide a link to the Creative Commons licence, and indicate if changes were made. The images or other third party material in this article are included in the article's Creative Commons licence, unless indicated otherwise in a credit line to the material. If material is not included in the article's Creative Commons licence and your intended use is not permitted by statutory regulation or exceeds the permitted use, you will need to obtain permission directly from the copyright holder. To view a copy of this licence, visit http://creativecommons.org/licenses/by/4.0/. 


\section{References}

1. Bilu, Yu., Hanrot, G., Voutier, P.M.: Eexistence of primitive divisors of Lucas and Lehmer numbers. With an appendix of M. Mignotte. J. Reine Angew. Math. 539, 75-122 (2001)

2. Bilu, Yu., Komatsu, T., Luca, F., Pizarro-Madariaga, A., Stănică, P.: On a divisibility relation for Lucas sequences. J. Number Theory 163, 1-18 (2016)

3. Carmichael, R.D.: On the numerical factors of the arithmetic forms $\alpha^{n} \pm \beta^{n}$. Ann. of Math. 15, 30-70 (1913)

4. Dujella, A., Pethô, A.: A generalization of a theorem of Baker and Davenport. Quart. J. Math. Oxford 49, 291-306 (1998)

5. Gómez Ruíz, C.A., Luca, F.: An exponential Diophantine equation related to the sum of powers of two consecutive $k$-generalized Fibonacci numbers. Colloq. Math. 137, 171-188 (2014)

6. Koshy, T.: Fibonacci and Lucas Numbers with Applications. Wiley, Hoboken (2001)

7. Laurent, M., Mignotte, M., Nesterenko, Yu.: Formes linéaires en deux logarithmes et déterminants d'interpolation. J. Number Theory 55, 285-321 (1995)

8. Luca, F., Oyono, R.: An exponential Diophantine equation related to powers of two consecutive Fibonacci numbers. Proc. Jpn. Acad. 137(Ser. A), 45-50 (2014)

9. Mignotte, M.: A kit for linear forms in three logarithms. Preprint (2008)

10. Rihane, S.E., Faye, B., Luca, F., Togbé, A.: An exponential Diophantine equation related to the difference between powers of two consecutive Balancing numbers. Ann. Math. Inf. 50, 167-177 (2019)

11. Rihane, S.E., Faye, B., Luca, F., Togbé, A.: On the exponential Diophantine equation $P_{n}^{x}+P_{n+1}^{x}=P_{m}$. Turk. J. Math. 43, 1640-1649 (2019)

Publisher's Note Springer Nature remains neutral with regard to jurisdictional claims in published maps and institutional affiliations. 